\title{
Local two-photon couplings and the $J=0$ fixed pole in real and virtual Compton scattering
}

\author{
Stanley J. Brodsky* \\ Theory Group, SLAC National Accelerator Laboratory, 2575 Sand Hill Road, 94025 Menlo Park, California, USA \\ Felipe J. Llanes-Estrada ${ }^{\dagger}$ \\ Departmento Física Teórica I, Facultad de Ciencias Físicas, Universidad Complutense de Madrid, 28040 Madrid, Spain
}

Adam P. Szczepaniak

Department of Physics and Nuclear Theory Center, Indiana University, Bloomington, Indiana 47405, USA

(Received 5 December 2008; published 20 February 2009)

\begin{abstract}
The local coupling of two photons to the fundamental quark currents of a hadron gives an energyindependent contribution to the Compton amplitude proportional to the charge squared of the struck quark, a contribution which has no analog in hadron scattering reactions. We show that this local contribution has a real phase and is universal, giving the same contribution for real or virtual Compton scattering for any photon virtuality and skewness at fixed momentum transfer squared $t$. The $t$ dependence of this $J=0$ fixed Regge pole is parameterized by a yet unmeasured even charge-conjugation form factor of the target nucleon. The $t=0$ limit gives an important constraint on the dependence of the nucleon mass on the quark mass through the Weisberger relation. We discuss how this $1 / x$ form factor can be extracted from high-energy deeply virtual Compton scattering and examine predictions given by models of the $H$ generalized parton distribution.
\end{abstract}

DOI: $10.1103 /$ PhysRevD.79.033012

PACS numbers: 13.40.Gp, 11.15.Tk, 11.55.Jy, 13.60.Fz

\section{INTRODUCTION AND OVERVIEW}

Exclusive hadron scattering processes at high energies are well described by the exchange of Pomeron and Regge exchanges. Regge theory, combined with the vector meson dominance model, provides a useful description of real and virtual high-energy photoproduction, single-photon processes which at the QCD level describe photon dissociation into quark-antiquark pairs which subsequently rescatter off the target constituents. Vector meson dominance and conventional Regge exchange, however, cannot account for contributions to real or virtual Compton scattering where two photons interact locally on the same quark of the target.

The local coupling of two photons to the fundamental quark current of a hadron leads to a contribution to the Compton amplitude of the form

$$
T_{\gamma^{*}(q) p \rightarrow \gamma^{*}\left(q^{\prime}\right) p^{\prime}}^{J=0}=-2 e^{2} F_{1 / x}^{C=+}(t) \boldsymbol{\epsilon} \cdot \boldsymbol{\epsilon}^{\prime},
$$

where $t=\left(p^{\prime}-p\right)^{2}$ is the square of the momentum transferred to the nucleon. The even charge conjugation " $1 / x$ " form factor $F_{1 / x}^{C=+}(t)$ is real for spacelike $t$. Unlike normal Regge exchange, contributions to the Compton amplitude which behave as $\beta_{R}(t) s^{\alpha_{R}(t)}$, the $J=0$ fixed-pole contribution is energy independent at any fixed $t$. Remarkably

\footnotetext{
*sjbth@slac.stanford.edu

ffllanes@fis.ucm.es

aszczepa@indiana.edu
}

$T^{J=0}$ is also independent of the incident and final photon virtualities $q^{2}$ and $q^{\prime 2}$ as well as the skewness $\xi=-\left(q^{2}+\right.$ $\left.q^{\prime 2}\right) / 4 p \cdot q$ for any given fixed $t$. It thus appears in real photon scattering as well as virtual Compton scattering. Because it has a real phase, the local contribution to virtual Compton scattering has maximal interference with the Bethe-Heitler bremsstrahlung contributions to $\ell p \rightarrow$ $\ell^{\prime} \gamma p$ [1,2]. These amplitudes can also be measured in timelike two-photon processes such as $\gamma^{*} \gamma^{*} \rightarrow H \bar{H}$ and $\gamma^{*} \rightarrow H \bar{H} \gamma$. Because the $J=0$ contribution arises from the local interactions of the two photons, there is no analog in any hadron scattering amplitude, and thus it cannot be obtained from models based on vector meson dominance. Unlike normal Regge trajectories, the local two-photon interaction only couples to scalar mesons in the $t$ channel, not a sum over states with progressively higher orbital angular momentum. The isospin of the contributing scalar mesons can be $I=0,1$, and 2. (However, no isospin-2 meson, an exotic by necessity, is currently well established).

In the case of the proton target, there are two $C=+$ amplitudes with the local $J=0$ structure: helicityconserving and helicity flip, analogous to the Dirac and Pauli form factors. The $F_{1 / x}^{C=+}(t)$ form factor for each quark flavor is obtained by summing over all quarks in the hadron weighted by $\sum e_{q}^{2}$. The integrand also contains an extra factor of $1 / x$ relative to the Dirac and Pauli electromagnetic form factor where $x$ is the usual light-front fraction $x=k^{+} / p^{+}=\left(k^{0}+k^{z}\right) /\left(p^{0}+p^{z}\right)$ of the quark in the 
hadron light-front wave function. Hence the name " $1 / x$ " form factor. It can also be related to the $1 / x$ moment of the $H$ and $E$ generalized parton distributions (GPDs), which parameterize deeply virtual Compton scattering $[3,4]$. The helicity-conserving form factor at $t=0$ is the $1 / x$ moment which appears in the Weisberger formula [5] for $\partial M^{2} / \partial m_{q}^{2}$. The two-photon form factors obtained from extracting the $J=0$ contribution to Compton scattering thus give new complimentary information on the structure of the target hadron.

The origin of the $J=0$ contribution is trivial in supersymmetric QCD where the electromagnetic current couples to charged scalar squarks. In this case the theory contains a local four-point interaction $e_{s}^{2} s^{\dagger} A^{\mu} A_{\mu} s$ where $s$ is the squark field. See Fig. 1. This "seagull" interaction couples the two photons locally to the hadron and gives a Born $J=0$ contribution to the Compton amplitude proportional to the charged squared of the squark. Since the four-point interaction is local, all radiative corrections from the strong interactions of the squarks are incorporated into the $C=+$ hadron form factors. The fact that a fixed pole can exist in the Compton amplitude $\gamma p \rightarrow \gamma p$ (real or virtual) due to the seagull interaction was first pointed out by Creutz [6].

In the case of spin- $1 / 2$ quarks, it is convenient to use the light-front Hamiltonian formulation of QCD [7]. The seagull interaction with scalar quarks is then replaced by the light-front instantaneous four-point interaction of the two photons with the quark current $e_{q}^{2} \bar{\psi} \gamma \cdot A\left(\gamma^{+} / i \partial^{+}\right) \gamma \cdot A \psi$. This interaction arises when one eliminates the constrained $\psi^{-}=\Lambda^{-} \psi$ quark field in light-cone gauge $A^{+}=0$. The same local two-photon interaction also emerges from the usual handbag Feynman diagram for Compton scattering. The numerator of the quark propagator $\gamma \cdot k_{F}+m$ appearing between the two photons in the handbag contributions to the Compton amplitude contains a specific term $\gamma^{+} \delta k^{-} / 2$ which cancels the $k_{F}^{2}-m^{2}$ Feynman denominator, leaving a local term inversely proportional to $k^{+}$,

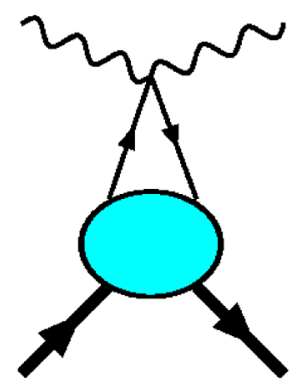

FIG. 1 (color online). The local coupling of two photons to a quark yields a fixed pole, a real and constant contribution to the Compton amplitude. In scalar electrodynamics this fixed pole is easy to recognize - it originates from the seagull coupling. For spin $1 / 2$ quarks, one needs to pick up an instantaneous (on the light-front) Z-diagram component of the handbag diagram, as shown in Eq. (6). equivalent to the light-front Hamiltonian contribution. Here $\delta k^{-} \equiv k_{F}^{-}-\left(k_{\perp}^{2}+m^{2}\right) / k^{+}=\left(k_{F}^{2}-m^{2}\right) / k^{+}$[see Eq. (6) below]. Thus in the spin- $1 / 2$ case, the two-photon interaction is local in impact space and light-front time $\tau=$ $x^{+}=x^{0}+x^{3}$, but it is nonlocal in the light-front coordinate $\sigma=x^{-}=x^{0}-x^{3}$. The $J=0$ contribution is intrinsic to the Feynman propagator; it is also essential for the gauge invariance of the Compton amplitude.

The $J=0$ fixed-pole contribution is well known in atomic physics since it gives the dominant contribution to high-energy elastic Compton scattering on an atom. In this case the seagull coupling $e^{2} \vec{A} \cdot \vec{A}^{\dagger} \phi \phi^{\dagger}$ to the nonrelativistic electron field of QED is responsible for the pointlike Thomson scattering on bound atomic electrons.

In principle, the $J=0$ contribution to Compton scattering measures the local coupling of photons to the fundamental carriers of the electromagnetic current at any photon resolution since it is independent of photon energy and virtuality. However, at finite energies the local contribution is screened by the contributions to the Compton amplitude from the remaining nonlocal interactions. For example, when the photon energy $\nu / M_{p}=$ $\left(s-M_{p}^{2}\right) / 2 M_{p}$ vanishes, there is no suppression from energy denominators and the nonlocal interactions give the contribution

$$
T_{\gamma p \rightarrow \gamma p}^{N L}(\nu \rightarrow 0)=-2\left[\left\langle\sum_{q} e_{q}\right\rangle^{2}-\left\langle\sum_{q} \frac{e_{q}^{2}}{x_{q}}\right\rangle\right] \boldsymbol{\epsilon} \cdot \boldsymbol{\epsilon}^{\prime}
$$

where the sum is over the quarks in the target and $x_{q}$ is the longitudinal momentum fraction carried by the quark of charge $e_{q}$ (in units of the proton electric charge). Thus at zero photon energy $s-M_{p}^{2} \rightarrow 0$, the local contribution,

$$
T_{\gamma p \rightarrow \gamma p}^{L}(\nu \rightarrow 0)=-2\left\langle\sum_{q} \frac{e_{q}^{2}}{x_{q}}\right\rangle \boldsymbol{\epsilon} \cdot \boldsymbol{\epsilon}^{\prime}
$$

is exactly canceled, and only the square of the total charge $e_{H}=\sum e_{q}$ appears in the Compton amplitude, $T=T^{L}+$ $T^{N L}$ :

$$
T_{\gamma p \rightarrow \gamma p}(\nu \rightarrow 0)=-2\left\langle\sum_{q} e_{q}\right\rangle^{2} \boldsymbol{\epsilon} \cdot \boldsymbol{\epsilon}^{\prime}
$$

consistent with the low-energy theorem. This cancellation of the local and nonlocal terms is demonstrated explicitly in Ref. [8]. Conversely, at higher energies, the nonlocal terms become suppressed or are strongly modified by the presence of energy denominators; this in principle, allows the local terms and their fundamental structure to emerge.

In the case of QCD, the contribution of the local coupling of the photons to quarks can be screened by the contributions from Pomeron exchange and other $C=+$ Reggeons which have intercepts $\alpha_{R}(0)>0$. These contributions, which have a complex phase dictated by analy- 
ticity and $t \leftrightarrow u$ crossing, will typically dominate the Compton amplitude at high energies. However, the energyand $q^{2}$-independent local contribution proportional to $\sum e_{q}^{2}$ is always present at any scale. For example, one can remove the Pomeron by measuring isovector channels such as the proton/neutron difference. At large $-t$, larger than some $-t_{0}$ determined by the Reggeon trajectory $\alpha_{R}(t)$ the nonsinglet $C=+$ Reggeons recede to negative values, $\alpha_{R}\left(-t>-t_{0}\right)<0$, exposing the $J=0$ contribution. In fact, the $s$-independent contribution $R_{V}(t)$ determined at Jefferson Laboratory [9] from the elastic Compton scattering amplitude at large $t$ can be identified with the $J=0$ contribution. If this identification is correct, the $R_{V}$ contribution to the Compton amplitude will be independent of photon virtuality at fixed large $t$ in $\gamma^{*} p \rightarrow \gamma p$ scattering.

In this paper we advocate the study of Compton scattering in the kinematical regime $s \gg-t>-t_{0}$ in order to extract the $J=0$ fixed-pole contribution as a fundamental test of QCD, and to measure the $1 / x$ form factor of the nucleon which parameterizes its $t$ dependence. In addition, if one studies deeply virtual Compton scattering, in the kinematical regime $s \gg Q^{2} \gg-t>-t_{0}$, one can express the form factor in terms of the generalized parton distribution function $H\left(x, \xi \sim Q^{2} / s \rightarrow 0, t\right)$, and learn about its $1 / x$ moment. The extrapolation to the forward limit $t \rightarrow$ $0^{-}$provides an important connection to the Weisberger relation, discussed in Sec. III B. We also note that the local two-photon coupling plays an implicit role in all inclusive processes involving two photons (or other vector fields) scattering or annihilating on a quark line such as $\gamma^{*} q \rightarrow$ $\gamma q$ and $\gamma \gamma \rightarrow q \bar{q}$.

The remainder of the paper is structured as follows. Section II gives a thorough analysis of the fixed pole and its parton interpretation. There we comment on pointlike scattering (Sec. II A), doubly virtual Compton scattering (Sec. II B), singly virtual Compton scattering (Sec. IID), and real Compton scattering (Sec. II E).

The forward limit of the fixed pole is presented in Sec. III, where we examine the range of values suggested by parton distribution functions in Sec. III A. The approach we follow is motivated by the procedure of Ref. [10] where a scalar-quark model, constrained to satisfy scaling relations and current conservation was applied to describe both real and imaginary parts of the $\gamma^{*} p \rightarrow \gamma^{*} p$ two-photon amplitude. In Sec. III B we review the Weisberger relation and the connection between the $1 / x$ moment of the parton disribution functions (pdf's) and the quark-mass dependence of the nucleon mass. At finite momentum transfer, the momenta of the two photons differ and the $1 / x$ form factor can be written as a moment of the generalized parton distribution. In Sec. IV we examine a few simple models of GPDs and conclude that the fixed pole is a general feature, revealed already by the valence part (three-quark component) of the nucleon's wave function, independently of whether traditional Regge theory is or not incorporated in a model. The common representation in terms of double distributions is briefly recalled in Sec. IVA. Then we provide an estimate of the $F_{1 / x}(t)$ form factor with valence quark model light-front wave functions in Sec. IV B. We examine the representation of the GPD in terms of the parton-proton scattering amplitude at large $t$ in Sec. IV C, and give a corresponding estimate for $F_{1 / x}(t)$.

Section $\mathrm{V}$ is dedicated to a preliminary examination of existing data sets. Early studies gave partial evidence for the pole in the forward [11] and in the off-forward [12] Compton amplitudes. We show that it is unlikely that current exclusive data has convincingly revealed the fixed-pole behavior, opening possibilities for the $12 \mathrm{GeV}$ Jefferson Laboratory facility or a proposed electron-ion collider. Conclusions and outlook are then presented in Sec. VI. We give a brief primer on the novel analytic properties of the fixed pole in Appendix A. The implications of isospin symmetry are reviewed in Appendix B.

\section{PARTON MODEL INTERPRETATION OF THE $J=0$ POLE IN TWO-PHOTON PROCESSES}

\section{A. Parton model and pointlike scattering}

The appearance of a $J=0$ pole in hadronic Compton processes originates from the local coupling of two photons with the quark constituents of the target hadron. We define the generalized Bjorken scaling variable

$$
\xi \equiv-\frac{q^{2}+q^{\prime 2}}{4 \nu}
$$

where $\nu=(s-u) / 4$. In the case of doubly virtual Compton scattering $q^{2}=q^{\prime 2}=-Q^{2}$ and $\xi$ becomes the Bjorken variable $x_{B}$, known from deep inelastic scattering (DIS), $x_{B} \equiv Q^{2} /(2 p \cdot q)$ with $p$ being the momentum of the target. In the case of singly virtual Compton scattering $q^{2}=-Q^{2}$ and $q^{\prime 2}=0, \xi$ becomes the symmetric scaling variable used in Ref. [13], and in this case it is related to the Bjorken variable $-x_{B}: \xi=x_{B} /\left(2-x_{B}\right)$. The physical interpretation of the $J=0$ pole is easiest to address in terms of the light-front coordinates $a^{\mu}=\left(a^{+}, a^{-}, a_{\perp}\right)$ with $a^{ \pm}=\left(a^{0} \pm a^{3}\right)$ in the frame where the + component of the incoming photon momentum vanishes, $q^{+}=0$. In this frame the light-front energy of the quark which is exchanged between the photons is given by either $k^{-}+q^{-}$ and $k^{\prime-}-q^{-}$for the $s$ and $u$ channel amplitude, respectively, both being of the order of $Q^{2} / \xi$. Thus the $\xi \rightarrow 0$ limit corresponds to the situation where the exchanged quark does not propagate over the light-front time.

Unlike the spin- 0 case, where the seagull contribution is explicitly local in all four space-time directions, the highenergy limit of a spin- $1 / 2$ exchange extends over the $t-z$, direction conjugated to the longitudinal moment $k^{+}$and $k^{\prime+}$. In the Bjorken limit virtual Compton amplitude, $\left(q^{2}=\right.$ $\left.-Q^{2}, q^{\prime 2}=0\right)$ is proportional to 


$$
\begin{aligned}
\frac{k+\not 1+m}{(k+q)^{2}-m^{2}+i \epsilon} & \rightarrow \frac{\gamma^{+}}{2 p^{+}}\left(\frac{\mathbf{1}}{\mathbf{x}}+\frac{\xi}{x} \frac{1}{x-\xi+i \epsilon}\right) \\
& =\frac{\gamma^{+}}{2 p^{+}} \frac{1}{x-\xi+i \epsilon}, \\
-\frac{k-\not q^{\prime}+m}{\left(k-q^{\prime}\right)^{2}-m^{2}+i \epsilon} & \rightarrow \frac{\gamma^{+}}{2 p^{+}}\left(\frac{\mathbf{1}}{\mathbf{x}}-\frac{\xi}{x} \frac{1}{x+\xi-i \epsilon}\right) \\
& =\frac{\gamma^{+}}{2 p^{+}} \frac{1}{x+\xi-i \epsilon},
\end{aligned}
$$

for direct and crossed-handbag, respectively, which are shown in Fig. 2. Here $x$ is the fraction of the incoming proton longitudinal momentum carried by the struck quark, $x=k^{+} / p^{+}$. In the right-hand side of each term we show the $1 / x$ piece which comes from canceling the $q^{-}$between numerator and denominator. As can be seen, for fixed $x$, upon taking the high-energy limit $\nu \rightarrow 0,(\xi \rightarrow 0)$ the remaining terms (from the on-shell numerator of the hard quark) cancel out as they are proportional to $\xi$. The behavior of the amplitude in the $x \rightarrow 0$ limit will be discussed in the following sections. One can immediately infer that in the high-energy limit both real and virtual Compton scattering on a quark contains the equivalent of the scalar seagull diagram for Dirac spin- $1 / 2$ fermions. This contribution reduces, at fixed $Q^{2}$, to $1 / x$, the fixed pole for elementary fermions and subsequently to the universal $1 / x$ form factor of the target.

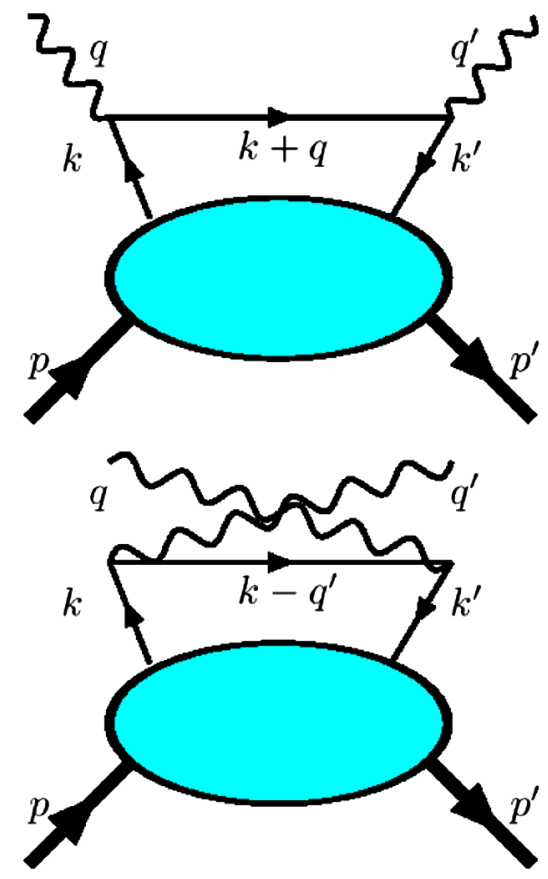

FIG. 2 (color online). The handbag and crossed-handbag diagrams convoluting the hard scattering amplitude together with a soft generalized parton distribution.

\section{B. $J=0$ pole in forward spatial doubly virtual Compton scattering}

The manner in which the $J=0$ pole manifests itself in the real and virtual Compton amplitude depends on the result of convolution with the parton distribution of the target hadron. The contribution from the $J=0$ pole to the doubly virtual Compton amplitude, $\gamma^{*}(q) p \rightarrow \gamma^{*}\left(q^{\prime}\right) p^{\prime}$, where both the initial and final state photons are spacelike, has been extensively studied in the past. Here we summarize the main results following Refs. [6,14].

In the forward case, i.e. $q=q^{\prime}, p=p^{\prime}$ doubly virtual Compton amplitude for a transverse photon, $\gamma^{*}(q) p \rightarrow$ $\gamma^{*}(q) p, T_{1}\left(Q^{2}, \nu\right)$,

$$
T_{\gamma^{*}(q) p \rightarrow \gamma^{*}(q) p}=\boldsymbol{\epsilon} \cdot \boldsymbol{\epsilon}^{\prime} T_{1}\left(Q^{2}, \nu\right)
$$

with $Q^{2}=-q^{2}, \nu=(s-u) / 4=p \cdot q . T$ is a an analytical function of $\nu$ except for cuts running along the real axis and starting at $\pm \nu_{t h}$. These assumptions and Cauchy's theorem lead to a dispersion representation,

$$
T_{1}\left(Q^{2}, \nu\right)=C_{\infty}+\frac{1}{\pi} \int_{\nu_{\mathrm{th}}^{2}}^{\infty} \frac{d \nu^{\prime 2}}{\nu^{\prime 2}-\nu^{2}-i \epsilon} \operatorname{Im} T_{1}\left(Q^{2}, \nu^{\prime}\right)
$$

where $C_{\infty}$ is the possible contribution from a part of the integration contour at infinity in the complex $\nu$ plane. Here we also used the crossing, $s-u$ symmetry relation $T_{1}\left(Q^{2}, \nu-i \epsilon\right)=T_{1}\left(Q^{2},-\nu+i \epsilon\right)$. In general, if $\operatorname{Im} T\left(Q^{2}, \nu\right) \propto \nu^{\alpha}$, as $\nu \rightarrow \infty$ the dispersive integral diverges and so does $C_{\infty}$. The sum, however, must be finite and equal to the physical amplitude. In a dispersive approach it is common to use subtractions to explicitly eliminate these infinities and any residual finite constants must be determined by comparison with experiment or derived from an effective theory. In the following we do not use the subtraction method, instead, since we are interested in the Bjorken limit we use the handbag diagram of Fig. 2 as representation of the amplitude. The handbag diagram, which follows from QCD, gives a finite physical amplitude, thus when written in terms of a Cauchy integral it corresponds to a dispersion relation with all subtractions determined by the parton distribution functions.

The spectral function $F_{1}\left(Q^{2}, \nu\right)=(1 / \pi) \operatorname{Im} T_{1}\left(Q^{2}, \nu\right)$ is nonzero for $2 \nu \geq 2 \nu_{\text {th }}=\left(M_{N}+m_{\pi}\right)^{2}+Q^{2}-M_{N}^{2}$. For real Compton scattering, with $Q^{2}=0$ it is convenient to subtract the dispersion relation at $\nu=0$ which eliminates the contribution from $C_{\infty}$ and replaces it by the known value of the amplitude $T_{1}(0,0)=-2$-the Thomson term. In the case of the forward doubly virtual Compton amplitude we can determine $C_{\infty}$ in the Bjorken limit. In terms of the Bjorken variable $x_{B}=Q^{2} / 2 \nu, T_{1}\left(x_{B}\right)=$ $\lim _{Q^{2} \rightarrow \infty} T_{1}\left(Q^{2}, \nu\right)$, Eq. (8) becomes $\left(x \equiv Q^{2} / 2 \nu^{\prime}\right)$

$$
T_{1}\left(x_{B}\right)=\Delta+T_{1, \text { handbag }}\left(x_{B}\right) \text {, }
$$

where we defined 


$$
T_{1, \text { handbag }}\left(x_{B}\right) \equiv \int_{0}^{1} d x \frac{2 x}{x_{B}^{2}-x^{2}-i \epsilon} f(x)
$$

and

$$
\Delta=T_{1}\left(x_{B}\right)-T_{1, \operatorname{handbag}}\left(x_{B}\right)=C_{\infty}+2 \int_{0}^{1} \frac{d x}{x} f(x) .
$$

Here and in the following $f(x)=(1 / \pi) \operatorname{Im} T_{1}(x)$ is the structure function. This structure function represents the sum over quark and antiquark distributions weighted with the parton's charge

$$
f(x)=e_{q}^{2}\left[f_{q}(x)+f_{\bar{q}}(x)\right] .
$$

The contribution to $T_{1}$ from the parton model is represented by the handbag diagram of Fig. 2 and is precisely given by $T_{1 \text {,handbag }}$ as discussed in Sec. II A. In Fig. 2 the blob represents strong parton-nucleon interactions and the upper part represents the hard scattering of virtual photons off a free quark (we ignore nonleading twist and perturbative QCD corrections).

In general, from phenomenological considerations, as well as from QCD evolution, it is expected that at small $x$ the structure function is given by

$$
f(x) \rightarrow f_{R}(x)=\sum_{\alpha} f_{R}^{\alpha}(x)=\sum_{\alpha} \frac{\gamma_{\alpha}}{x^{\alpha}}
$$

with $\alpha$ both positive and negative being the intercept of the Regge trajectory, $\alpha=\alpha(t=0)$; Pomeron exchange with $\alpha \sim 1$ and $t$-channel meson Regge trajectories with $\alpha \sim$ 0.5 are clearly visible in the data. Contributions from daughter trajectories and/or valence quarks typically have $\alpha<0$. A physical interpretation of the Regge contributions to hadron structure functions is discussed in Ref. [15].

Furthermore, in the Bjorken limit, deeply inelastic scattering is best interpreted as if the parton model gives the entire contribution to $T_{1}$, i.e.

$$
\Delta=0 \text {. }
$$

It thus follows that the Regge contribution to $T_{1}$ is given by

$$
\begin{aligned}
T_{1, R}\left(x_{B}\right) & =\int_{0}^{1} d x \frac{2 x}{x_{B}^{2}-x^{2}-i \epsilon} f_{R}(x)= \\
& =-\pi \sum_{\alpha} \frac{1+e^{-i \pi \alpha}}{\sin \pi \alpha} f_{R}^{\alpha}\left(x_{B}\right)+T_{1, R}^{J=0}+T_{1, R}^{J<0}\left(x_{B}\right)
\end{aligned}
$$

where

$$
\begin{aligned}
T_{1, R}^{J=0} & =2 \sum_{\alpha} \frac{\gamma_{\alpha}}{\alpha} \\
T_{1, R}^{J<0}\left(x_{B}\right) & =2 \sum_{\alpha} \sum_{J=-2,-4, \cdots} \frac{\gamma_{\alpha}}{\alpha-J} \frac{1}{x_{B}^{J}} .
\end{aligned}
$$

Note that there is no constant contribution to the structure function since the phase of an $\alpha=0$ contribution to the forward Compton amplitude is real.

We have grouped terms according to their importance at high energies (small $x_{B}$ ). The $J=0$ contribution corresponds to the $T_{1, R}^{J=0}$ term and $T_{R}^{J<0}$ represents subleading terms at high energies.

Even though there is a $J=0$ pole at the level of elementary interaction between photons and quarks, originating from the $1 / x$ term in the photon-quark scattering amplitude, it could be the case that the convolution of the elementary amplitude with the structure functions removes the $J=0$ pole in the full amplitude. This could happen if

$$
\sum_{\alpha} \frac{\gamma_{\alpha}}{\alpha}=0
$$

Thus pointlike interactions are necessary but not sufficient for the existence of a $J=0$ pole. However, since the Regge contributions are $t$ dependent, $\alpha=\alpha(t)$, this accidental cancellation would only occur at one value of $t$.

For a general structure function $f(x)$, the small- $x$ behavior is carried by the Regge-type $f_{R}^{\alpha>0}(x)=\sum_{\alpha>0} \gamma_{\alpha} / x^{\alpha}$ that we have already analyzed, such that

$$
\lim _{x \rightarrow 0}\left[f(x)-f_{R}^{\alpha>0}(x)\right]=0 .
$$

After isolating the Regge and non-Regge parts the $T_{1}$ amplitude can be written as

$T_{1}\left(x_{B}\right)=-\pi \sum_{\alpha>0} \frac{1+e^{-i \pi \alpha}}{\sin \pi \alpha} f_{R}^{\alpha>0}\left(x_{B}\right)+T_{1}^{J=0}+T_{1}^{J<0}\left(x_{B}\right)$,

where

$$
\begin{aligned}
T_{1}^{J=0}= & -2 \int_{0}^{1} \frac{d x}{x} f_{v}(x)+2 \sum_{\alpha>0} \frac{\gamma_{\alpha}}{\alpha} \\
T_{1}^{J<0}\left(x_{B}\right)= & x_{B}^{2} \int_{0}^{1} \frac{d x}{x} \frac{2}{x_{B}^{2}-x^{2}-i \epsilon} f_{v}(x) \\
& +2 \sum_{\alpha>0} \sum_{J=-2,-4, \cdots} \frac{\gamma_{\alpha}}{\alpha-J} \frac{1}{x_{B}^{J}},
\end{aligned}
$$

and we have defined the valence structure function as

$$
f_{v}(x) \equiv f(x)-f_{R}^{\alpha>0}(x)
$$

so that by construction $f_{v}(0)=0$. It thus follows that at high energies $T_{1}^{J>0}\left(x_{B} \rightarrow 0\right)$ vanishes. In the forward direction it is the first term on the right-hand side which dominates and grows with energy as $1 / x_{B}^{\alpha}$.

\section{Nonforward, spatial doubly virtual Compton scattering}

The $T_{1}$ amplitude at finite momentum transfer $t=\left(p^{\prime}-\right.$ $p)^{2}, \gamma^{*}(q) p \rightarrow \gamma^{*}\left(q^{\prime}\right) p^{\prime}$ can be obtained from analyticity in $t$, which implies analyticity in $\alpha$ since $\alpha=\alpha(t), f(x) \rightarrow$ $f(x, t)$ (which will be interpreted shortly as a generalized 
parton distribution) with

$$
f_{R}^{\alpha}(x, t)=\frac{\gamma(t)}{x^{\alpha(t)}} .
$$

Since Regge trajectories $\alpha(t)$ have positive slope, as $-t$ increases they will reach some $-t \leq-t_{0}$ where all $\alpha(t)$ become negative. There is partial evidence of this from Ref. [16]. At this point the amplitude becomes dominated by the contribution by the $J=0$ pole.

\section{D. $\boldsymbol{J}=\mathbf{0}$ pole in virtual Compton scattering}

The main focus of deeply virtual Compton scattering (DVCS) $\gamma^{*}(q) p \rightarrow \gamma\left(q^{\prime}\right) p^{\prime}$ measured in $e p \rightarrow e^{\prime} p^{\prime} \gamma X$ at large $q^{2}$ and the production of real photons $q^{12}=0$ is the measurements of the generalized parton distributions, which depend on longitudinal quark momenta and momentum transfer.

However, DVCS also allows a new window into the study of the local $J=0$ fixed-pole contribution which we have emphasized provides a fundamental test of QCD. Furthermore since the $J=0$ contribution has a real phase, it has maximal overlap with Bethe-Heitler bremmstralung contribution to $e p \rightarrow e^{\prime} p^{\prime} \gamma X[1,2]$.

As before we work in the Bjorken limit, with $Q^{2}, \nu \rightarrow \infty$ with $x_{B}=Q^{2} /(2 p \cdot q)$ finite and $-t / Q^{2} \rightarrow 0$. For simplicity we consider the nucleon helicity-nonflip amplitude, which dominates in the forward limit, and write a fixed- $t$ dispersion relation in $\nu$,

$$
\nu=\frac{s-u}{4}=\frac{4 p \cdot q-Q^{2}}{4}=\frac{Q^{2}}{4 \xi} .
$$

We recall from Sec. II A that $\xi$ plays the role of a generalized Bjorken variable for DVCS. Using the $s-u$ crossing symmetry of $T_{1}$, we have

$$
\begin{aligned}
T_{1}\left(Q^{2}, \nu, t\right)= & C_{\infty}(t)+\frac{1}{\pi} \int_{\nu_{t h}^{2}}^{\infty} \frac{d \nu^{\prime 2}}{\nu^{\prime 2}-\nu^{2}-i \epsilon} \\
& \times \operatorname{Im} T_{1}\left(Q^{2}, \nu^{\prime}, t\right) .
\end{aligned}
$$

In the Bjorken limit we expect $T_{1}$ to be dominated by the handbag diagram of Fig. 2. The connection between dispersion relation representation and the QCD asymptotic handbag picture was also discussed in [17-19]. In analogy to doubly virtual Compton scattering we rewrite the dispersion relation for $T_{1}$ at fixed $t$ in terms of dimensionless variables $\xi$ and $x=Q^{2} / 4 \nu^{\prime}$ in the scaling limit as

$$
T_{1}(\xi, t)=C_{\infty}(t)+\frac{\xi^{2}}{\pi} \int_{0}^{1} \frac{2 d x}{x} \frac{\operatorname{Im} T_{1}(x, t)}{\xi^{2}-x^{2}-i \epsilon} .
$$

Determining the subtraction constant, which will turn out to be the fixed-pole contribution, requires additional information. Again, this is done by assuming QCD handbag dominance in the Bjorken limit. If we assume scaling and handbag dominance valid for $Q^{2} \gg-t>-t_{0}$ with momentum transfer such that Regge intercepts, $\alpha(t)<0$ are negative,

$T_{1}(\xi, t)=-\int_{-1}^{1} d x H(x, \xi, t)\left[\frac{1}{x+\xi-i \epsilon}+\frac{1}{x-\xi+i \epsilon}\right]$.

From it we can read that at high energies

$$
C_{\infty}(t)=\lim _{\xi \rightarrow 0} T_{1}(\xi, t)=-2 \int_{-1}^{1} d x \frac{H(x, 0, t)}{x} .
$$

At large $-t,-t>-t_{0}, H(x \rightarrow 0,0, t) \rightarrow 0$ and the integral is finite and real. At $\xi=0$ the $H(x, 0, t)$ defines the generalized parton distribution functions discussed in Sec. II B

$$
H(x, 0, t)=\theta(x) f_{q}(x, t)-\theta(-x) \bar{f}_{q}(-x, t)
$$

with the two functions referring to the quark and antiquark distributions, respectively. At $t=0$ they reduce to the parton distribution functions measured in deep inelastic scattering. In Sec. II B the single pdf $f(x, t)$ was used to denote the net contribution from quark and antiquarks of given flavor. It is related to the two pdf's given above by

$$
f(x, t)=f_{q}(x, t)+\bar{f}_{q}(x, t), \quad x>0 .
$$

We can also obtain the imaginary part of $T_{1}$ from Eq. (26) to read

$$
\frac{1}{\pi} \operatorname{Im} T_{1}(\xi, t)=H(\xi, \xi, t)-H(-\xi, \xi, t) .
$$

Substituting Eq. (27) and (30) in Eq. (25) we obtain the analog of Eq. (9),

$$
\begin{aligned}
T_{1}(\xi, t)= & -2 \int_{-1}^{1} d x \frac{H(x, 0, t)}{x}+\xi^{2} \int_{-1}^{1} \frac{d x}{x} \\
& \times \frac{H(x, x, t)-H(-x, x, t)}{\xi^{2}-x^{2}-i \epsilon} .
\end{aligned}
$$

We can also write $T_{1}$ as

$$
\begin{aligned}
T_{1}(\xi, t)= & \int_{-1}^{1} d x \frac{x}{\xi^{2}-x^{2}-i \epsilon} H^{+}(x, x, t) \\
& +\int_{-1}^{1} \frac{d x}{x}\left[H^{+}(x, x, t)-H^{+}(x, 0, t)\right],
\end{aligned}
$$

where we defined the positive charge conjugation, $\mathrm{H}^{+}$ generalized parton distributions,

$$
H^{+}(x, x, t) \equiv H(x, x, t)-H(-x, x, t)
$$

and

$$
\begin{aligned}
H^{+}(x, 0, t) & =H(x, 0, t)-H(-x, 0, t) \\
& =\theta(x) f(x, t)-\theta(-x) f(-x, t)
\end{aligned}
$$

with $f(x, t)$ given by Eq. (29). As long as $-t>-t_{0}$ with all Regge intercepts negative $\alpha(t)<0, H^{+}(x, x, t)$ vanishes in the limit $x \rightarrow 0$ and the $J=0$ pole contribution to $T_{1}$ is given by 


$$
\begin{aligned}
T_{1}^{J=0}(t) & =\lim _{\xi \rightarrow 0} T_{1}(\xi, t)=-\int_{-1}^{1} \frac{d x}{x} H^{+}(x, 0, t) \\
& =-2 \int_{-1}^{1} \frac{d x}{x} H(x, 0, t) .
\end{aligned}
$$

Now we are ready to lift the assumption that $-t>t_{0}$ and consider the situation where some of the intercepts are positive, $\alpha(t)>0$, still assuming Bjorken scaling. Since $T_{1}$ is finite for any $\xi$ it follows from Eq. (32) that

$$
\lim _{x \rightarrow 0}\left[H^{+}(x, x, t)-H^{+}(x, 0, t)\right]=0 .
$$

Thus we define the valence part $H_{v}$ as the part of $H$ which is finite in the $x \rightarrow 0$ limit,

$$
H_{v}(x, x, t) \equiv H(x, x, t)-H_{R}(x, t)
$$

which implies $H_{v}^{+}(x, x, t) \equiv H_{v}(x, x, t)-H_{v}(-x, x, t)$ and similarly, following Eq. (36)

$$
H_{v}(x, 0, t) \equiv H(x, 0, t)-H_{R}(x, t)
$$

and $H_{v}^{+}(x, 0, t) \equiv H_{v}(x, t)-H_{v}(-x, t)$ which are all finite in the limit $x \rightarrow 0$. Here we assumed that in general the small- $x$ behavior is of the Regge type given by

$$
\begin{aligned}
& H_{R}(x, t) \equiv \theta(x) \sum_{\alpha>0} \frac{\gamma_{\alpha}(t)}{x^{\alpha(t)}}-\theta(-x) \sum_{\bar{\alpha}>0} \frac{\bar{\gamma}_{\bar{\alpha}}(t)}{(-x)^{\bar{\alpha}(t)}}, \\
& H_{R}^{+}(x, t) \equiv H_{R}(x, t)-H_{R}(-x, t) .
\end{aligned}
$$

Finally for the amplitude $T_{1}$ we obtain

$$
\begin{aligned}
T_{1}(\xi, t)= & \int_{-1}^{1} d x \frac{x}{\xi^{2}-x^{2}-i \epsilon} H_{v}^{+}(x, x, t) \\
& +\int_{-1}^{1} d x \frac{x}{\xi^{2}-x^{2}-i \epsilon} H_{R}^{+}(x, t) \\
& +\int_{-1}^{1} \frac{d x}{x}\left[H_{v}^{+}(x, x, t)-H_{v}^{+}(x, 0, t)\right] .
\end{aligned}
$$

To extract the $J=0$ pole contribution we need to study the $\xi \rightarrow 0$ limit. In this limit we find

$$
\begin{aligned}
\lim _{\xi \rightarrow 0} T_{1}(\xi, t)= & -\int_{-1}^{1} \frac{d x}{x} H_{v}^{+}(x, 0, t) \\
& +\lim _{\xi \rightarrow 0} \int_{-1}^{1} d x \frac{x}{\xi^{2}-x^{2}-i \epsilon} H_{R}^{+}(x, t) .
\end{aligned}
$$

The second term on the right-hand side in Eq. (41) yields terms which grow with energy as $\sim 1 / \xi^{\alpha(t)},(\alpha(t)>0)$, a constant term $O\left(\xi^{0}\right)$, and subleading terms which decrease with increasing energy, $O\left(\xi^{2-\alpha(t)}\right)$. Extracting the constant term from the Regge term we obtain for the $J=0$ contribution to $T_{1}$,

$$
\begin{aligned}
T_{1}^{J=0}(t)= & -2 \int_{-1}^{1} \frac{d x}{x} H_{v}(x, 0, t)+2 \sum_{\alpha>0} \frac{\gamma_{\alpha}(t)}{\alpha(t)} \\
& +2 \sum_{\bar{\alpha}>0} \frac{\bar{\gamma}_{\bar{\alpha}(t)}}{\bar{\alpha}(t)} .
\end{aligned}
$$

We note that since $H_{v}(x, 0, t)$ is the generalized parton distribution, the expression for the $J=0$ pole contribution in DVCS with real photon in the final state is exactly the same as obtained in Sec. II B for doubly virtual Compton scattering.

We have thus shown that the $J=0$ pole contribution is universal, i.e. the same for doubly virtual Compton scattering, as given by Eq. (20) and in virtual Compton scattering, as given by Eq. (42). Again, as the momentum transfer increases, the intercepts $\alpha$ become negative and the virtual Compton amplitude at high energies $\xi \rightarrow 0$ becomes dominated by the $J=0$ pole contribution, while other details of nucleon structure described by the valence generalized parton distribution $H_{v}(x, \xi, t)$ are suppressed by powers of $\xi^{1-\alpha(t)}$ where $\alpha(t)<0$ is the largest intercept.

Bjorken scaling in DVCS has been demonstrated to hold $[20,21]$ from an analysis of QCD corrections to the elementary two-photon parton amplitudes. In particular it has been shown that IR divergences can be absorbed into the soft parton-nucleon amplitudes parameterized by the generalized parton distributions. This proof, however, relies on the assumption that GPD's are finite at the break points, i.e.

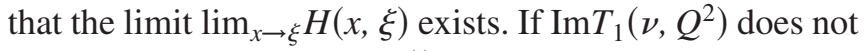
scale i.e. $\operatorname{Im} T_{1}\left(\nu, Q^{2}\right) \sim \nu^{\alpha(t)}$ with $\alpha(t)>0$ at small $t$ then in the Bjorken limit $\operatorname{Im} T_{1}\left(\nu, Q^{2}\right)$ diverges and so does $\lim _{\xi \rightarrow \xi} H^{+}(\xi, \xi)=\infty$. The converse is also true, in such a case one would expect $T_{1}\left(\nu, Q^{2}\right) \sim\left(Q^{2} / \xi\right)^{\alpha(t)}$. A possibility of nonscaling in DVCS and its consequences for the high-energy behavior has been studied in Ref. [22] and shown to be consistent with scaling observed in doubly virtual Compton amplitude. We note, however, that the $J=0$ contribution, being $\nu$ independent is truly universal, regardless of the scaling properties of $T_{1}$.

\section{E. Real Compton scattering (RCS)}

The first evidence for the existence of a $J=0$ contribution in the real Compton amplitude was developed by Damashek and Gilman, based on the dispersion theory and measurements of the total photoabsorption cross section $\sigma(\gamma p \rightarrow X)$.

The Gell-Mann, Goldberger, Thirring subtracted dispersion relation for the forward helicity no-flip Compton amplitude $T_{1}(\nu)=T_{1}\left(\nu, Q^{2}=0\right)$ is

$$
T_{1}(\nu)=T_{1}(0)+\frac{\nu^{2}}{\pi} \int_{\nu_{0}}^{\infty} \frac{d \nu^{\prime 2}}{\nu^{\prime 2}-\nu^{2}-i \epsilon} \frac{\operatorname{Im} T_{1}\left(\nu^{\prime}\right)}{\nu^{\prime 2}},
$$

where $\nu=(s-u) / 4$ and $\nu_{0}$ corresponds to the pion production threshold, $\nu_{0}=m_{\pi} M_{N}+m_{\pi}^{2} / 2$.

As noted earlier, in the low-energy limit, $\nu \rightarrow 0$, the Compton amplitude has local and nonlocal contributions [cf. Eqs. (2) and (3)]

$$
T_{1}(0)=T_{1}^{L}+T_{1}^{N L},
$$

and $T_{1}(0)=-2$ is the Thomson term. Note that $T_{1}^{N L}$ 
includes the contributions of the cat's ears diagrams where the incident photon couples to one quark current and the outgoing photon couples to the other current. The local term $T_{1}^{L}$ is real; only the nonlocal contribution has an absorptive, imaginary part. For RCS we can define $x \equiv$ $\nu_{0} / \nu$ so the amplitude parallels that of virtual Compton scattering,

$$
T_{1}(x)=T_{1}(0)+\frac{1}{\pi} \int_{0}^{1} d x^{\prime} \frac{2 x^{\prime}}{x^{2}-x^{\prime 2}-i \epsilon} \operatorname{Im} T_{1}\left(x^{\prime}\right) .
$$

Subtracting the Regge contribution and defining

$$
f_{v}(x) \equiv \frac{1}{\pi} \operatorname{Im} T_{1}(x)-\sum_{\alpha \geq 0} \frac{\gamma_{\alpha}}{x^{\alpha}}
$$

gives for the energy independent $J=0$ contribution,

$$
T_{1}^{J=0}=T_{1}(0)-2 \int_{0}^{1} \frac{d x}{x} f_{v}(x)+2 \sum_{\alpha>0} \frac{\gamma_{\alpha}}{\alpha} .
$$

At high energy, $\nu \rightarrow \infty, x \rightarrow 0$, the quark-photon interactions are universal, and we thus expect the $J=0$ contribution to be identical to the local $T_{1}^{L}$ term,

$$
T_{1}^{J=0}=T_{1}^{L} .
$$

In the limit $t \rightarrow 0$ this relates the normalization of the $1 / x$ moment of the forward RCS amplitude to that of the DVCS in the limit $-t \rightarrow 0$,

$$
\begin{aligned}
& \int_{0}^{1} \frac{d x}{x} f_{v}\left(x, Q^{2} \rightarrow \infty, t=0\right) \\
& =1+\int_{0}^{1} \frac{d x}{x} f_{v}\left(x, Q^{2}=0, t=0\right) .
\end{aligned}
$$

For finite momentum transfer $t \neq 0$ the constant on the right-hand side should be replaced by $-1 / 2$ of the finite- $t$ subtraction constant from Eq. (43), which for fixed-angle scattering is expected to fall off as a power of $-t$, and one would expect the $1 / x$ moment in DVCS and RCS be identical at large $s$ and large $-t$, with $-t / s \sim O(1)$

$$
\int_{0}^{1} \frac{d x}{x} f_{v}\left(x, Q^{2} \rightarrow \infty, t\right)=\int_{0}^{1} \frac{d x}{x} f_{v}(x, 0, t) .
$$

Damashek and Gilman have used the dispersion relation in Eq. (43) and the measured photoabsorption cross section to determine $\operatorname{Re} T_{1}(\nu)$. They fit the high-energy photoabsorption cross section to $s$-channel resonances at low energies and the Pomeron $\alpha_{P}(0)=1$ and Reggeon $\alpha_{R}(0)=1 / 2$ contributions at high energies: $\sigma=\sum_{i} c_{i} \nu^{\alpha_{i}(0)-1}$. Since the Pomeron contribution has an imaginary phase, this form predicts a Reggeon $\nu^{\alpha_{i}(0)}=\nu^{1 / 2}$ contribution at high energy for the forward amplitude, which as we have argued is associated specifically with $T_{1}^{N L}(\nu)$. However, Damashek and Gilman also find that the dispersion relation predicts an additional constant contribution to $\operatorname{Re} T_{1}(\nu)$ at high energies. As we discussed above this can be identified with the local term $T_{1}^{L}$. Since this constant term is found empirically to have approximately the same value as the Born term, this implies

$$
-2 \int_{0}^{1} \frac{d x}{x} f_{v}(x)+2 \sum_{\alpha>0} \frac{\gamma_{\alpha}}{\alpha} \sim 0
$$

and that the $J=0$ fixed pole on the proton at $t=0$ has the value $-2\left\langle\sum_{q} e_{q}^{2} / x_{q}\right\rangle \sim-2\left\langle\sum_{q} e_{q}\right\rangle^{2}=-2$ in the energy domain of the photoabsorption experiment. An interesting test of this analysis would be the measurement of the photoabsorption cross section on a neutron target. One predicts a fixed pole a factor of $2 / 3$ smaller [see Eq. (89) below].

\section{PHENOMENOLOGY OF THE $J=0$ POLE}

\section{A. The $J=0$ pole in the forward limit and the structure function parametrization}

The $J=0$ pole in the forward limit $t=0$ is given in terms of parton distribution functions measurable in DIS. Parton distribution functions need to diverge at small $x$ due to the Regge behavior of hadron scattering amplitudes and simple statistical arguments. This well-known observation, supported by the extensive HERA data at small $x$, was made by Kuti and Weisskopf [23]. The Lorentz invariant phase space of a parton whose transverse momentum can be ignored is $d p_{i} / 2 E_{i}=d x / 2 x$. If one ignores dynamical effects, and imposes the statistical hypothesis that all states for the sea partons are equally likely, one sees that the sea distribution functions scale as

$$
\lim _{x \rightarrow 0} f_{s}(x)=\frac{\gamma_{1}}{x} .
$$

The small $x$ divergence in the sea part of the pdf reflects simply large phase space available at large energy. Kuti and Weisskopf further observed that the Regge behavior of the photon-proton scattering amplitude needs to stem from Regge behavior of the parton distribution functions within the proton and found that the small $x$ behavior if pdf's is given by

$$
\lim _{x \rightarrow 0} f(x)=\frac{\gamma_{\alpha}}{x^{\alpha(0)}}
$$

with $\alpha(0)$ the usual intercept of the Regge trajectory with the $t=0$ axis in a Chew-Frautschi plot. As discussed in the previous section the pdf's with $\alpha<0$ are to be associated with the valence distributions, and $\alpha>0$ with the sea distribution. The split between sea and valence Regge contribution supports the interpretation that pointlike current interactions on target constituents is dual to exchanges of all residual or daughter Regge trajectories.

Modern fits to deep inelastic scattering data routinely employ a small $Q^{2}$ parameterization of the pdf's which is a simple variation of the Kuti-Weisskopf statistical model, namely [24] (see Table I in that paper for the parameters $A_{i}$, $\left.\eta_{i}, \lambda_{i} \epsilon_{i}, \gamma_{i}\right)$ 


$$
x f_{i}(x)=A_{i} x^{\eta_{i}}(1-x)^{\lambda_{i}}\left(1+\epsilon_{i} \sqrt{x}+\gamma_{i} x\right) .
$$

We expose its Regge form around $x=0$ by expanding the $(1-x)$ in powers of $x$,

$$
\begin{aligned}
f_{i}(x)= & A\left(x^{\eta_{i}-1}+\epsilon_{i} x^{\eta_{i}-1 / 2}+\left(\gamma_{i}-\lambda_{i}\right) x^{\eta_{i}}\right. \\
& \left.-\epsilon_{i} \lambda_{i} x^{\eta_{i}+1 / 2}-\gamma \lambda x^{\eta_{i}+1}+\ldots\right) .
\end{aligned}
$$

In terms of these MRST [24] parameters, the Regge intercept is $\alpha(0)=1-\eta_{i}$. Phenomenology of deep inelastic scattering requires $\eta$ to be smaller than 1 for several pdf's. For the valence flavors, a typical Regge intercept is of order $\alpha(0)=1 / 2$. This is the case for the GRV98 pdf set [25] that has exponents $\alpha(0)=-0.85$ and -0.52 for the light sea and valence pdf's, respectively. (Here "valence" is used in the sense of Ref. [24].) Notice that the $\sqrt{x}$ in Eq. (53) gives rise to subleading Regge power laws. For the MRST98 [24] pdf sets, an also widely used alternative, the power-law exponents have higher variation around classical Regge theory and the $u$ proton's valence component has a somewhat high intercept $\alpha_{u}(0) \in(0.53,0.59)$, the $d$ valence component being definitely at odds with other phenomenology with $\alpha_{d}(0) \simeq 0.73$ as large as the sea component. The subleading Regge behavior is also given by the $\sqrt{x}$ factor in Eq. (53), and having an intercept larger than zero, it also causes a divergence. In both GRV98 and MRST98 sets the gluon pdf behaves as a valencelike parton with a very small intercept at this low scale, an indication of the gluon degrees of freedom being gapped at low energy [26].

Since $T_{1}^{J=0}$ was initially assessed in Ref. [10] there has been immense experimental progress and accurate pdf fits for a wide range of $Q^{2}$ are now available. We have been conservative and chosen a decade-old set of fit functions which have been thoroughly tested.

In Tables I and II we have collected the values of the integrals in Eq. (58) multiplied by the corresponding quark to positron charge ratios $e_{q}^{2}$. We have explicitly separated the contribution from quark and antiquark and subtracted all Regge poles with $\alpha>0$ from $f, \bar{f}$ to yield $f_{v}$ and $\bar{f}_{v}$ (which are finite at $x=0$ ). This is also equal to Eq. (20) up to a $(-1 / 2)$ factor.

The sum over the entries in a column in this table should be a number of $O(1)$, judging by the results of Ref. [11]. This relies on a cancellation which does not occur using the current parameterizations of pdfs. This possibly signals a systematic uncertainty in the way these parameterizations are written down. As can be seen the results are spread over an order of magnitude, and in some cases even the sign is difficult to ascertain. The reason is simple. The regulated integral over the valence pdf's is perfectly well behaved. However, subleading Regge poles with an intercept just below zero, which are integrable and need no subtraction, will yield a non-negligible contribution. The MRST and even the GRV fit which seem to comply better with the theoretical arguments of Kuti and Weisskopf, employ a
TABLE I. $\quad F^{C=+}(0)$ defined in Eq. (1), that is $-\frac{1}{2} T_{1}^{J=0}=$ $\int_{0}^{1} \frac{d x}{x}\left(f(x)-f^{R}(x)\right)-\sum_{\alpha>0} \frac{\gamma_{\alpha}}{\alpha}$ for MRST98 [24] and GRV [25] full parton distribution functions. We take both sets at the low scale defining the fit parameters since we prefer analytical expressions to better control the subtractions. These scales are $1 \mathrm{GeV}^{2}$ for the MRST set and $0.26(0.4) \mathrm{GeV}^{2}$ for the leading order (next-to-leading order) GRV set. The latter has no strange sea component at this low scale. A large spread in the results comes from the uncertainty in the subtraction constants $\gamma / \alpha$, which are not yet very well determined.

\begin{tabular}{lccccc}
\hline \hline Quark & $\begin{array}{c}\text { MRST } \\
\text { low } \\
\text { gluon }\end{array}$ & $\begin{array}{c}\text { MRST } \\
\text { central } \\
\text { gluon }\end{array}$ & $\begin{array}{c}\text { MRST } \\
\text { upper } \\
\text { gluon }\end{array}$ & LO GRV & NLO GRV \\
\hline$u$ & 51 & 14 & 13 & -36 & 17 \\
$\bar{u}$ & -5.3 & -1.3 & -7.0 & 62 & 9.7 \\
$d$ & 6.1 & 5.9 & 5.0 & -120 & -11 \\
$\bar{d}$ & -0.78 & -0.46 & -1.8 & -62 & -13 \\
$s$ & -1.5 & -0.43 & -2.2 & 0 & 0 \\
$\Sigma$ & 50 & 18 & 7.0 & -160 & 2.7 \\
\hline \hline
\end{tabular}

formula such as Eq. (53). There, the subleading terms with $\sqrt{x}$ and $x$ are phenomenologically added to improve the fit to structure function data. For most applications the precise power law at small $x$ of $f$ is not needed; a computer code which yields $f$ as usually provided, suffices, but to have the best possible fits becomes critical if we enhance the small $x$ part by computing the $1 / x$ moment. This can be seen by considering the following toy distribution function:

$$
f(x)=x^{\alpha}(1-x)(1+\alpha)(2+\alpha) .
$$

The expectation value of the momentum fraction for the parton is

$$
\langle x\rangle=\frac{1+\alpha}{3+\alpha},
$$

which converges to $1 / 3$ in the $\alpha \rightarrow 0$ limit, but then

$$
\left\langle\frac{1}{x}\right\rangle=\left(1+\frac{2}{\alpha}\right)
$$

which is divergent in the same limit. Therefore we see how it is really critical to control Regge poles with intercept near and below zero. Indeed this is not the case for the standard pdf parameterizations, and while a group of trajectories bunch around intercept values 0.4 to 0.6 , others are well below zero.

Another comment is in order: the recent G0 and Happex experiments at Jefferson Lab [27] have not yet settled what level of strange sea is needed to account for parity violation even at small $Q^{2}$. Therefore, one should take the GRV fit where the strange sea vanishes with caution. Another difference between the MRST and the GRV fits worth recalling is that the isospin asymmetry disappears in the Regge limit of the simplest MRST set we employ, not so in the GRV fits where it is controlled by a standard Reggeon of intercept $\simeq 1 / 2$. Although not much can be said from 
Table I, it seems that the integral over the valence pdf's is negative.

\section{B. The $1 / x$ moment and Weisberger's relation}

The importance of the $1 / x$ moment of parton distribution functions, which measures the valence quark contribution to the $T_{1}^{J=0}$ amplitude, was stressed by Weisberger in Ref. [5]. There he derived a relation between this $1 / x$ moment and the derivative of the squared proton mass with respect to the squared parton mass taking into account only the kinetic energy dependence on the quark mass. The resulting relation is valid for the parton mass and the corresponding distribution function defined at the same scale $\mu$. In modern notation and normalization [24], Weisberger's result reads

$$
\frac{\delta M_{N}^{2}}{\delta m_{i}^{2}(\mu)}=\frac{1}{e_{i}^{2}} \int_{0}^{1} \frac{d x}{x}\left[f_{v, i}(x)_{\mu}+\bar{f}_{v, i}(x)_{\mu}\right] .
$$

Here $f_{v, i} / e_{q}^{2}$ is the $i$ th-valence quark distribution function, and likewise for the antiquark (once the Regge part has been subtracted). This relation can be easily understood following the formal argument of Weisberger. He observed that the shift of the nucleon's energy $E$ upon shifting the energy of a parton $E_{i}$ in the Bjorken limit is given by

$$
\delta E=\frac{1}{e_{i}^{2}} \int_{0}^{1} d x_{i} f_{v, i}\left(x_{i}\right) \delta E_{i}\left(x_{i}\right) .
$$

In this limit parton momentum is taken to be parallel to that of the nucleon, $p_{i}=x_{i} P$, and for the parton with mass $m_{i}$ one has,

$$
E_{i}^{2}=m_{i}^{2}+x_{i}^{2} P^{2}
$$

so that

$$
2 E_{i} \delta E_{i}=\delta\left(m_{i}^{2}\right) \quad \text { or } \quad \delta E_{i}=\frac{\delta\left(m_{i}^{2}\right)}{2 x_{i} P} .
$$

Identifying $E$ with nucleon mass and using $\delta M^{2}=2 E \delta E$ we find

$$
\delta M^{2}=\delta E^{2}=\frac{1}{e_{i}^{2}} \int_{0}^{1} d x_{i} f_{v, i}\left(x_{i}\right) \delta m_{i}^{2} \frac{2 E}{2 P x_{i}}
$$

from which Eq. (58) follows. On first impression one would think of taking also a derivative of the pdf respect to the quark mass, but this is not the case according to the Hellman-Feynman theorem.

One can see that Weisberger's result holds simply by noting that in light-front quantization the Hamiltonian contains a kinetic energy term

$$
M_{\mathrm{kin}}^{2}=\sum_{i} \frac{k_{\perp}^{2}+m_{i}^{2}}{x_{i}}
$$

and no other explicit quark-mass dependence. Upon taking the expectation value $\left\langle\delta M^{2} / \delta m_{i}^{2}\right\rangle$ in the nucleon state and, in the collinear approximation, ignoring the $k_{\perp}$ immedi- ately leads to Weisberger relation of Eq. (58). In light-front QCD there is one further implicit quark-mass dependence in the quark-gluon vertex. This is analogous to the QED case, where the spin-flip vertex term $e \rightarrow e \gamma$ in the QED light-front Hamiltonian is proportional to $m_{e}$ (e.g. Table 6 on page 78 of Ref. [7]). This yields an additional contribution to the Weisberger relation.

The Weisberger relation involves the proton state, which by definition is normalizable and therefore contains only bound constituents; i.e., any contribution to the structure functions which can be interpreted as originating from processes in which photon splits into the $q \bar{q}$ pair which later rescatter off protons' constituents should not be included in Eq. (58). Of course since we do not know a proton's wave function and pdf's are known through fits to data rather than from first principle calculations, the separation of the types of processes is at best phenomenological. Since the left-hand side in the Weisberger relation is finite, the valence structure function entering the righthand side has to satisfy $\lim _{x \rightarrow 0} f_{v, i}(x)=0$. We thus take the structure function to be given by Eq. (21), and the values of $\delta M_{N}^{2} / \delta m_{i}^{2}$ obtained for different pdf parameterizations are given in Table I.

\section{THE $1 / x$ FORM FACTOR AND OFF-FORWARD $J=0$ POLE}

In the previous section we considered the $J=0$ component of the $T_{1}$ amplitude in the forward limit of doubly virtual Compton scattering and its relation to the Weisberger sum rule. Now we return to finite momentum transfer, where the $t$-dependence of the fixed pole $C_{\infty}(t)$ provides a new form factor of the nucleon.

In analogy with the conventional Dirac form factor expressed in terms of the generalized parton distribution

$$
F(t)=\int_{-1}^{1} d x H(x, 0, t) .
$$

TABLE II. As in Table I but without the $\sum_{\alpha>0} \frac{\gamma_{\alpha}}{\alpha}$ terms, that is, corresponding to Eq. (58). Again systematic differences appear between the MRST and GRV sets, but the different MRST parameterizations are now very consistent.

\begin{tabular}{lccccc}
\hline \hline $\begin{array}{l}\text { Quark } \\
\text { flavor }\end{array}$ & $\begin{array}{c}\text { MRST } \\
\text { low } \\
\text { gluon }\end{array}$ & $\begin{array}{c}\text { MRST } \\
\text { central } \\
\text { gluon }\end{array}$ & $\begin{array}{c}\text { MRST } \\
\text { upper } \\
\text { gluon }\end{array}$ & LO GRV & NLO GRV \\
\hline$u$ & -6.7 & -7.0 & -11 & 12 & 10.6 \\
$\bar{u}$ & -20 & -16 & -20 & -12 & -12 \\
$\delta M_{N}^{2}$ & -27 & -23 & -31 & $\simeq 0$ & -1.1 \\
$\delta m_{u}^{2}$ & -39 & -52 & -39 & 120 & 130 \\
$\bar{d}$ & -27 & -27 & -33 & 67 & 70 \\
$\delta M_{N}^{2}$ & -66 & -79 & -72 & 180 & 200 \\
$\delta m_{d}^{2}$ & -15 & -22 & -29 & 0 & 0 \\
$\delta S M_{N}^{2}$ & -31 & -45 & -58 & 0 & 0 \\
$\overline{\delta m_{s}^{2}}$ & $\simeq 600$ & $\simeq-350$ & $\simeq 1500$ & 4.4 & 12 \\
$g$
\end{tabular}


At sizable $-t$ when Regge intercepts are negative, the $1 / x$ form factor defined in Eq. (1) is given by [cf. Eq. (35)]

$$
F_{1 / x}(t)=\int_{-1}^{1} \frac{d x}{x} H(x, 0, t)
$$

and for small $t$ where there can be Reggeons with intercept $\alpha>0$ according to Eq. (42) we find,

$$
F_{1 / x}(t) \equiv \int_{-1}^{1} \frac{d x}{x} H_{v}(x, 0, t)-\sum_{\alpha>0} \frac{\gamma_{\alpha}}{\alpha(t)}-\sum_{\bar{\alpha}>0} \frac{\bar{\gamma}_{\bar{\alpha}}}{\bar{\alpha}(t)} .
$$

As momentum transfer increases in virtual Compton scattering the energy dependent part of the transverse photon amplitude decreases at high energies and $F_{1 / x}(t)$ is expected to dominate the cross section. As discussed in Sec. II D the $J=0$ pole contribution to the transverse amplitude in singly virtual Compton scattering is given by the $J=0$ component of the doubly virtual amplitude.
There has been much progress in the extraction of the conventional Dirac form factor using lattice QCD [28] thanks to the use of twisted boundary conditions; in principle the $F_{1 / x}$ can also be extracted. Meanwhile we can provide an estimate using models of generalized parton distributions. We restrict ourselves to three simple models: ansatz in terms of double distributions [29-32], the lightfront wave function representation in the valence constituent quark model of the proton [33], and the quark-diquark model [34]. We comment on how the $J=0$ fixed-pole behavior is universal and arises for any reasonable model of the GPD's-as it should.

\section{A. Double distribution parametrization of GPD's}

In this ansatz GPD's are computed in the symmetric frame through

$$
\begin{aligned}
H^{q}(x, \xi, t)= & \frac{F_{1}^{q}(t)}{F_{1}^{q}(0)}\left(\frac{\theta(\xi+x)}{1+\xi} \int_{0}^{\min \{(x+\xi / 2 \xi),(1-x / 1-\xi)\}} d y F^{q}\left(x_{+}, y\right)-\frac{\theta(\xi-x)}{1+\xi} \int_{0}^{\min \{(\xi-x / 2 \xi),(1+x / 1-\xi)\}} d y F^{\bar{q}}\left(x_{-}, y\right)\right) \\
& +\theta(\xi-|x|) D(x / \xi, t) / N_{f} .
\end{aligned}
$$

In the original parametrization without a $D$-term, $t$ dependence is factorized and parameterized in terms of the proton's $F_{1}$ form factor, which is usually taken as a dipole

$$
F_{1}(t)=\frac{1}{\left(1-\frac{t}{0.7 \mathrm{GeV}^{2}}\right)^{2}}\left[1-\frac{t \mu}{4 M_{N}^{2}}\right]
$$

with $\mu \simeq 2.793$ being the anomalous magnetic moment. The $x_{ \pm}$variables in Eq. (65) are defined by

$$
x_{+}=\frac{x+\xi-2 \xi y}{1+\xi}, \quad x_{-}=\frac{\xi-x-2 \xi y}{1+\xi} .
$$

Without the $D$-term, the momentum-transfer dependence of the $1 / x$ will thus be the same as $F_{1}(t)$

$$
\begin{aligned}
F_{1 / x}^{q}(t) & =\int_{-1}^{1} \frac{d x}{x} H(x, \xi=0, t) \\
& =F_{1}(t) \int_{-1}^{1} \frac{d x}{x} H(x, \xi=0,0) .
\end{aligned}
$$

We note that this will be the same in any factorizable ansatz for the generalized parton distributions. In the limit $\xi \rightarrow 0$ the first and second term on the right-hand side of Eq. (65) contribute to Eq. (68). The last term, in Eq. (65), the so called $D$-term, [30], gives a contribution to $H$ which depends on the fraction $y \equiv x / \xi$. It leads to a contribution to the DVCS amplitude which is part of the fixed pole and remains finite and real in the $s \rightarrow \infty(\xi \rightarrow 0)$ limit. Its contribution to the $F_{1 / x}$ form factor is given by

$$
F_{1 / x}^{D}(t)=F_{1 / x}^{D}(0)=\frac{1}{N_{f}} \int_{-1}^{1} \frac{d y}{y} D(y, t) .
$$

It is worth noting that the fixed pole receives contribution from the valence part of the quark double distribution, as well as the $D$-term.

\section{B. Light-front constituent quark wave functions}

Following Ref. [33] we write the light-front valence constituent quark model representation of the GPD. If higher Fock space components of the proton are suppressed, the only interval where $H(x, \xi ; t)$ is nonvanishing is $\xi<x<1$, whereas the particle-number changing contribution in the interval $-\xi<x<\xi$ and the antiparticle contribution in $-1<x<-\xi$ are absent

$$
\begin{aligned}
H(x, \xi, t)= & \frac{\theta(x-\xi)}{(1-\xi)} \sum_{\lambda_{i}} \int \prod_{i=1}^{3}\left(\frac{d x_{i} d^{2} \mathbf{k}_{\perp i}}{16 \pi^{3}}\right) \\
& \times 16 \pi^{3} \delta\left(1-x_{1}-x_{2}-x_{3}\right) \delta^{(2)}\left(\sum \mathbf{k}_{\perp i}\right) \\
& \times \delta\left(x-x_{1}\right) \psi_{\lambda^{\prime}=+}^{*}\left(x_{i}^{\prime}, \mathbf{k}_{\perp i}^{\prime}, \lambda_{i}^{\prime}\right) \\
& \times \psi_{\lambda=+}\left(x_{i}, \mathbf{k}_{\perp i}, \lambda_{i}\right) .
\end{aligned}
$$

Here the light-front quark momentum fractions in the final proton are $x_{i}^{\prime}=\left(x_{i}(1+\xi)-2 \xi\right) /(1-\xi)$ for the struck quark and $x_{i}^{\prime}=x_{i}(1+\xi) /(1-\xi)$ for spectators, respectively. A simple Gaussian model is commonly used [35] for the light-front valence wave function. Here we consider

$$
\psi_{3}\left(x_{i}, \mathbf{k}_{i}\right)=A e^{b^{2}\left[M^{2}-\sum_{i=1}^{3}\left(m^{2}+\mathbf{k}_{\perp}^{2} / x\right)_{i}\right]} .
$$

The parameters, which are listed in Table III together with a model for the running $\alpha_{s}$ with the UV scale $\Lambda$ and IR 
BRODSKY, LLANES-ESTRADA, AND SZCZEPANIAK

TABLE III. A workable parameter set for the Gaussian valence quark model.

\begin{tabular}{lcc}
\hline \hline Description & Variable & Value \\
\hline Running of $\alpha_{s}$ & $\Lambda$ & $205 \mathrm{MeV}$ \\
Freezing of $\alpha_{s}$ & $M_{0}$ & $1.05 \mathrm{GeV}$ \\
Gaussian falloff & $b$ & 4.85 \\
Quark mass & $m$ & $270 \mathrm{MeV}$ \\
\hline \hline
\end{tabular}

freezing mass $M_{0}$,

$$
\alpha_{s}\left(p^{2}\right)=\frac{4 \pi}{\left(9 \log \left(\left(p^{2}+M_{0}^{2}\right) / \Lambda^{2}\right)\right)},
$$

provide a reasonable fit to the proton form factor [36], which is shown in Fig. 3. These wave functions, however, are too soft to be used in conjunction with an impulse approximation for DVCS at sufficiently large $t$, were the $J=0$ can be extracted experimentally. This is illustrated in Fig. 4 where it is seen how the magnetic form factor in the impulse approximation with these soft wave functions departs from its experimental value already at momentum transfer of the order of $3 \mathrm{GeV}^{2}$. Still it is illustrative as a benchmark, and probably not too misleading in the interval $1<-t<3 \mathrm{GeV}^{2}$, to plot the $1 / x$ from factor, $F_{1 / x}(t)$. This is shown in Fig. 5. Should one wish to extend the computation to somewhat higher $-t$ without abandoning the impulse approximation, a hard component with a power-law falloff would have to be included in the wave function. Reasonable power-law models are available in the literature [37-39].

It is worth making some generic observations about the valence quark model. The first is that the Compton ampli-

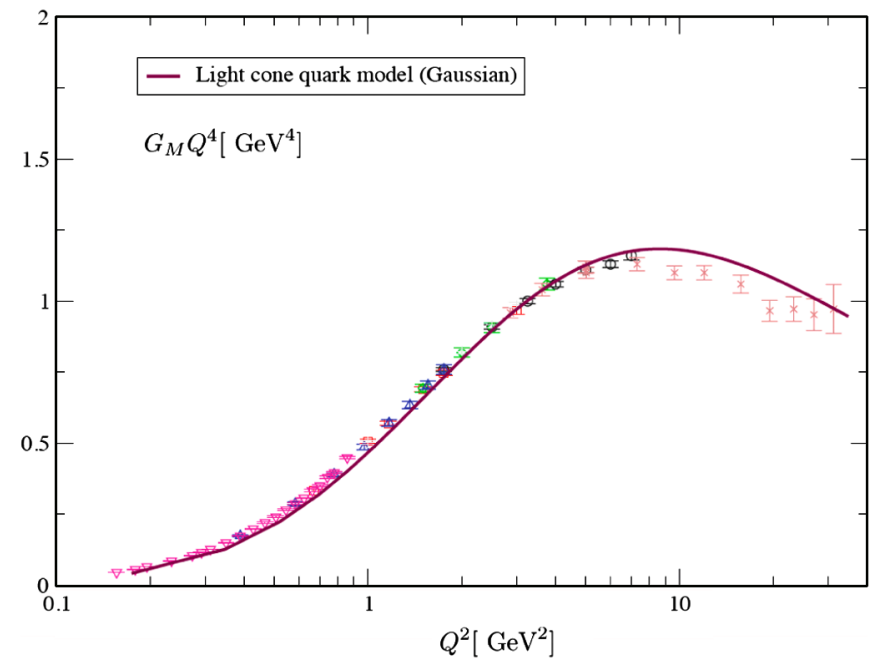

FIG. 3 (color online). Proton Sachs form factor in the impulse approximation with light-front Gaussian wave function of Eq. (71) and one-gluon exchange. The parameters employed are $m=250 \mathrm{MeV}$ and $b=1.2 \mathrm{GeV}^{-2}$. Data are taken from Ref. [61]
PHYSICAL REVIEW D 79, 033012 (2009)

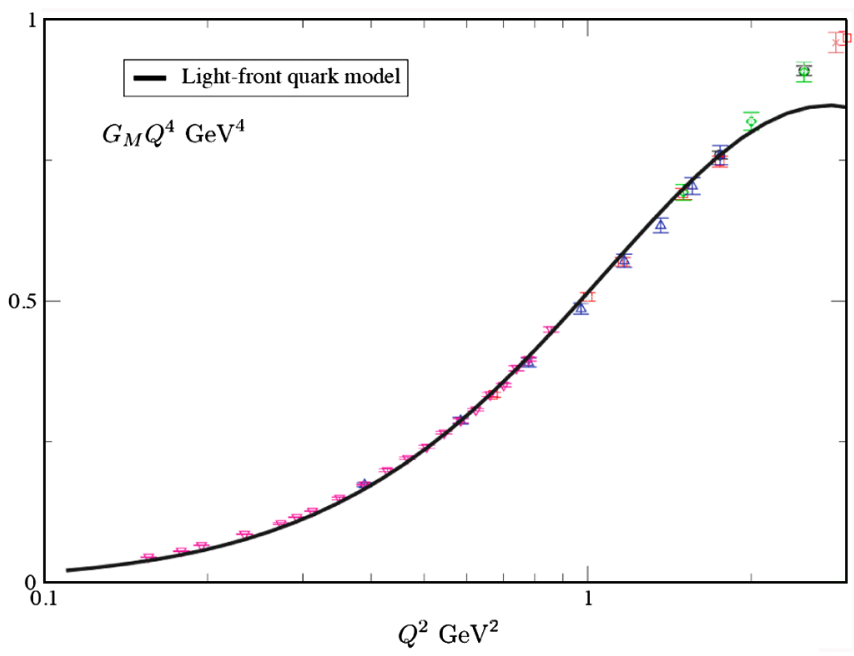

FIG. 4 (color online). Proton Sachs form factor in the impulse approximation with light-front Gaussian wave function from Eq. (71). Since this approximation excludes the hard, one-gluon exchange component (as opposed to Fig. 3), the form factor representation can only be trusted until about $3 \mathrm{GeV}^{2}$.

tude has no imaginary part at leading order and leading twist, because $H$ is real, and in the model, at the break points $x=-\xi$ and $x=\xi, H(\xi, \xi, t)$ and $H(-\xi, \xi, t)$ vanish. Finiteness of the GPD at the break points is a generally assumed feature. This behavior is reminiscent of, for example, the pion distribution amplitude at the end points, and indeed arises from the same underlying assumption, namely, that the end-point region (or break-point region in the case of GPD's) is governed by the one-gluon-exchange evolution. This may not necessarily be the case that was discussed, for example, in Ref. [40] and its consequences for DVCS in Ref. [22].

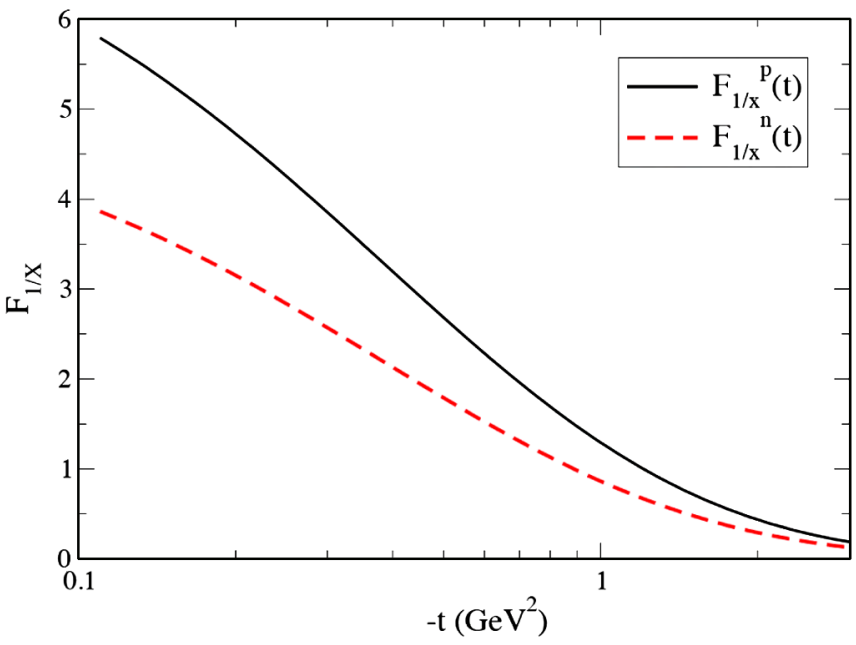

FIG. 5 (color online). The $1 / x$ form factor of the proton and neutron in the valence light-front quark model. The ratio is equal to $2 / 3$ arising from the ratio of quark electric charges. Parameters are as in Fig. 3. 


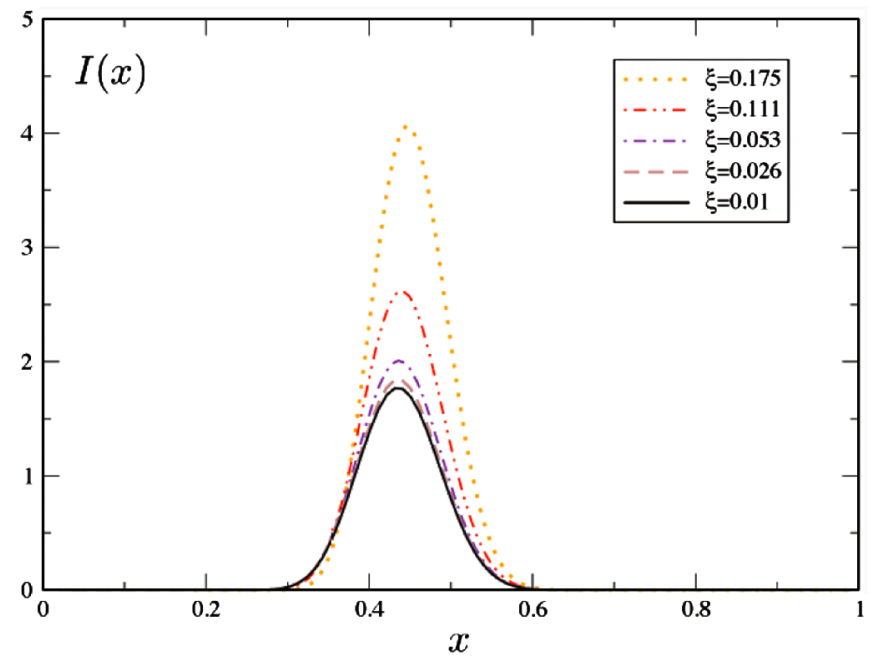

FIG. 6 (color online). We plot the unintegrated handbag amplitude given in Eq. (73). The area under the left-most curve is smaller than all others, and tags the $J=0$ fixed pole.

Second, valence models do not include large- $x$ tails of the sea quarks. The contribution of the sea quarks have been modeled, for example, in Ref. [35,41], and the model seems to fare well when compared with data available at current kinematics. However, the lack of a usable dynamical calculation of the sea quark component of the proton wave function makes the number of ad hoc parameters increase with each new Fock subspace added.

Still with our simplified valence version of the model we can see how the $J=0$ fixed pole arises here too. In Fig. 6 we plot the unintegrated handbag amplitude

$$
I(x) \equiv H(x, \xi, t)\left(\frac{1}{x-\xi}+\frac{1}{x+\xi}\right)
$$

whose integral over $x$ yields the Compton amplitude. [The relation between this $H$ GPD and that in the asymmetric frame as defined in Refs. [34,42] is $(1+$ $\xi) H(x, \xi, t)=H\left(x_{\text {asym }}, \zeta, t\right)$.] As can be seen from the figure, the area below the curve does not tend to zero in any limit. The minimum of these areas is an irreducible contribution to the Compton amplitude, independent of the skewness. It can be extracted by going to the smallest possible skewness experimentally achievable. We choose $t=-0.5 \mathrm{GeV}^{2}$ for the plot, where the fast exponential drop with $t$ has not yet set in, but this property of there being a finite area independent of the skewness is not a $t$ artifact.

A more sophisticated fit to all available form factor data has been carried on by Diehl et al. [43]. The fit includes Dirac and Pauli form factors and a careful study of the propagation of their errors. However, the authors run into the Regge behavior of the pdf's at small $x$ and therefore can only plot the $F_{1 / x}$ at sizeable $t$. We adapt their computation in Fig. 7.

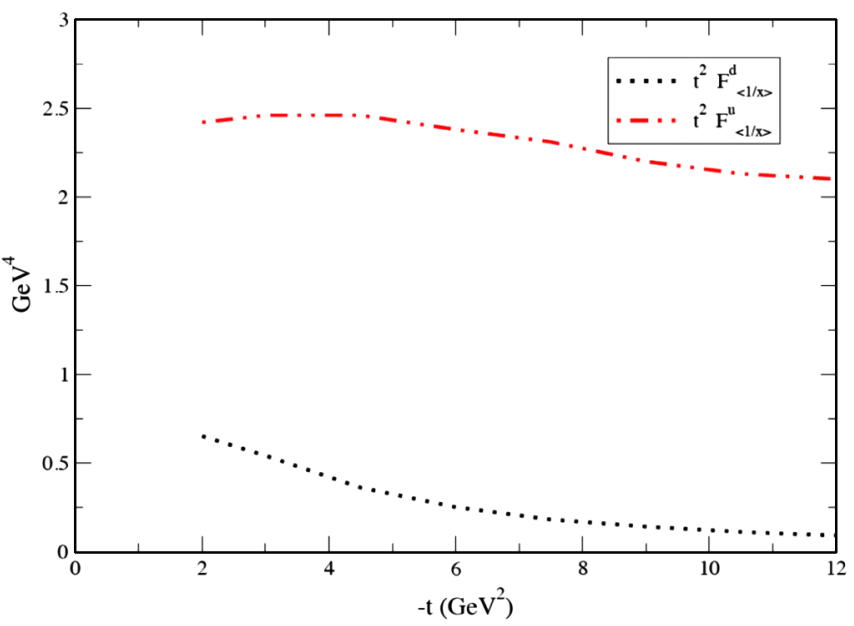

FIG. 7 (color online). An evaluation of the $1 / x$ form factors of the proton has been carried out in the Gaussian light-front constituent quark model, valid for large $-t$. The authors present the flavor-separated form factors from a set of GPD's fit to a number of conventional Dirac and Pauli form factors. Computer data from Ref. [43].

\section{GPD in the covariant parton-nucleon model}

The covariant parton model of Ref. [44] was extended to Compton scattering and DVCS in Ref. [10]. Since this model has not been widely used in the past, we will rely on it more extensively for our application, as a contribution to the current discussion on GPD's. The idea is to construct a model of the quark-parton scattering amplitude, with unamputated quark legs of momentum $k, k^{\prime}$ and Dirac indices $\alpha, \beta$ and two amputated proton legs with momentum $p, p^{\prime}$ and helicities $\lambda, \lambda^{\prime}$. Since the parton legs are off shell, this amplitude is a function of four different Lorentz scalars, that can be chosen as the three Mandelstam invariants $\hat{s}=\left(p+k^{\prime}\right)^{2}, t=\hat{t}=\left(k^{\prime}-k\right)^{2}=\left(p^{\prime}-p\right)^{2}$, $\hat{u}=(p-k)^{2}$, and $k^{2}$. The squared momentum of the returning parton can be expressed as $k^{12}=s+t+u-$ $2 M_{N}^{2}-k^{2}$. We will denote this amplitude by $T_{\lambda \lambda^{\prime} ; \alpha \beta}\left[\hat{s}, t, \hat{u}, k^{2}\right]$. Once the dependence on nucleon helicity has been factored out, the reduced amplitude can be expanded in the basis of Dirac matrices in the parton indices multiplied by scalar functions of the partonnucleon Mandelstam variables. Eventually the $H$ generalized parton distribution can be expressed in terms of such scalar functions,

$$
H(x, \xi, t)=x p^{+} \int \frac{d^{4} k}{(2 \pi)^{4}} \delta\left(x p^{+}-k^{+}\right) T\left[\hat{s}, t, \hat{u}, k^{2}\right]
$$

where $p^{+}$is the longitudinal momentum of the target. For example, a model in which the parton-nucleon amplitude is taken to be described to be an $\hat{u}$ - channel exchange of a diquark of mass $\lambda$ would correspond to 


$$
T\left[\hat{s}, t, \hat{u}, k^{2}\right]=\left(i g\left(k^{2}\right)\right) \frac{1}{(p-k)^{2}-\lambda^{2}+i \epsilon}\left(i g\left(k^{\prime 2}\right)\right)
$$

with vertex functions $g\left(k^{2}\right)$ describing off-shell partons. These are expected to become perturbative at large parton virtuality $k^{2}$ and at small virtuality are expected to be soft e.g. determined by the constituent quark mass, $m_{q}$. These features can be incorporated by writing a dispersion representation for $g\left(k^{2}\right)$ in the form

$$
g\left(k^{2}\right)=\int_{0}^{\infty} d \Lambda^{2} \frac{\rho\left(\Lambda^{2}\right)}{k^{2}-\Lambda^{2}+i \epsilon}
$$

with the spectral function given by

$$
\rho\left(\Lambda^{2}\right)=g m_{q}^{4} \frac{d}{d \Lambda^{2}} \delta\left(\Lambda^{2}-m_{q}^{2}\right) .
$$

The support of the resulting GPD's, as shown below, is the standard $-1<x<1$ region which arises in a perturbative analysis where the parton-nucleon vertex function has no structure. In the DIS limit, where one can think of partons as essentially free, this is probably a good approximation, although it has been speculated in Ref. [45] which at the limited $Q^{2}$ accessible to experiment, one might find GPD's with support outside of these nominal limits, which is due to the structure in the $k^{-}$plane stemming from the vertex functions. Given that solid theory would rely on controlling the effect of quark confinement on the analytical structure of vertex functions, we ignore this possibility here but leave the question open. Consider therefore for now the model defined by Eqs. (75) and (76). We employ the variable $\tilde{k}^{-}=P^{+} k^{-}$so that an inverse power of $P^{+}$comes out of the $k^{-}$integral and yields

$$
\begin{aligned}
H(x, \xi, t)= & (x+\xi) \int_{0}^{\infty} \frac{d\left|\mathbf{k}_{\perp}\right|^{2}}{2(2 \pi)^{4}} \\
& \times \int_{0}^{2 \pi} d \phi_{\perp} \int_{0}^{\infty} d \tilde{k}^{-} T\left[\hat{s}, t, \hat{u}, k^{2}\right]
\end{aligned}
$$

in place of Eq. (74) and with $k^{+}, k^{\prime}$ fixed as above. Finally, we introduce both $\hat{s}$ and $\hat{u}$ channel amplitudes with a relative factor $\gamma$. That is, we generalize Eq. (75) to

$$
\begin{aligned}
\frac{T\left(\hat{s}, t, \hat{u}, k^{2}, k^{\prime 2}\right)}{\left(i g\left(k^{2}\right)\right)\left(i g\left(k^{\prime 2}\right)\right)}= & \frac{1}{(p-k)^{2}-\lambda^{2}+i \epsilon} \\
& +\frac{\gamma}{\left(p^{\prime}+k\right)^{2}-\lambda^{2}+i \epsilon} .
\end{aligned}
$$

The crossed, $\hat{s}$-channel term is necessary because of the known forward relation yielding conventional antiquark pdf's $H(x<0, \xi=0, t=0)=-\bar{q}(-x)$. The $\hat{u}$-channel amplitude yields a nonvanishing $H(x)$ for $x>\xi$ only [34]. Since the valence part of the antiquark distribution functions are known to be much smaller than the quark pdf's, the factor $\gamma$ accounts for suppression of the $\hat{s}$-channel. We recall that the $1 / x$ from factor, which we are interested in is defined through the valence region. If we were to write a general representation for $T$ containing the sea contributions in the low- $x$ region the $\hat{s}$ and $\hat{u}$ channel terms would become constrained by crossing symmetry.

The parton-proton amplitude so defined is a holomorphic function of $\tilde{k}^{-}$and has four poles, three in each of the $s$ and $u$ channels, whose positions are depicted in Fig. 8. The $\hat{u}$-channel diquark propagator yields a simple pole denoted as $\kappa_{1}^{-}$and given by the condition $\hat{u}-\lambda^{2}+i \epsilon=$ 0 . The vertex functions yield two double poles, respectively, denoted by $\kappa_{2}^{-}$for $k^{2}-\Lambda^{2}+i \epsilon=0$ and $\kappa_{3}^{-}$for $k^{\prime 2}-\Lambda^{2}+i \epsilon=0$. Finally the $s$-channel pole from $s-$ $\lambda^{2}+i \epsilon=0$ is denoted $\kappa_{4}^{-}$. After deforming the $\tilde{k}^{-}$integral, picking up the poles and performing the differentiation with respect to $\Lambda$ [c.f. Eq. (76)] on both propagators independently we obtain [46]

$$
\begin{aligned}
H(-\xi<x<\xi, \xi, t)= & -g^{2} m_{q}^{8}(x+\xi) \int_{0}^{\infty} \frac{d\left|\mathbf{k}_{\perp}\right|^{2}}{2(2 \pi)^{3}} \int_{0}^{2 \pi} d \phi_{\perp}\left[\frac{1}{1+x}\left(\frac{\gamma}{\left(k^{2}-m_{q}^{2}\right)^{2}\left(k^{\prime 2}-m_{q}^{2}\right)^{2}}\right)_{k^{-}=\kappa_{4}^{-}}\right. \\
& \left.+\left(\frac{1}{x+\xi}\right)^{2} \frac{1}{\left(\left(k^{\prime 2}-m_{q}^{2}\right)_{k^{-}=\kappa_{2}^{-}}\right.}\left[\frac{x-1}{\left(u-\lambda^{2}\right)^{2}}-\frac{\gamma(1+x)}{\left(s-\lambda^{2}\right)^{2}}+\frac{2(x-\xi)}{k^{\prime 2}-m_{q}^{2}}\left(\frac{1}{u-\lambda^{2}}-\frac{\gamma}{s-\lambda^{2}}\right)\right]_{k^{-}=\kappa_{2}^{-}}\right]
\end{aligned}
$$

and

$$
\begin{gathered}
H(\xi<x<1, \xi, t)=g^{2} m_{q}^{8}(x+\xi) \int_{0}^{\infty} \frac{d\left|\mathbf{k}_{\perp}\right|^{2}}{2(2 \pi)^{3}} \int_{0}^{2 \pi} d \phi_{\perp}\left(\frac{1}{\left(k^{\prime 2}-\Lambda^{2}\right)^{2}} \frac{1}{\left(k^{2}-\Lambda^{2}\right)^{2}}\right)_{k^{-}=\kappa_{1}^{-}} \frac{1}{1-x} \\
H(-1<x<-\xi), \xi, t)=g^{2} m_{q}^{8}(x+\xi) \int_{0}^{\infty} \frac{d\left|\mathbf{k}_{\perp}\right|^{2}}{2(2 \pi)^{3}} \int_{0}^{2 \pi} d \phi_{\perp}\left(\frac{1}{\left(k^{\prime 2}-\Lambda^{2}\right)^{2}} \frac{1}{\left(k^{2}-\Lambda^{2}\right)^{2}}\right)_{k^{-}=\kappa_{4}^{-}} \frac{1}{1+x},
\end{gathered}
$$




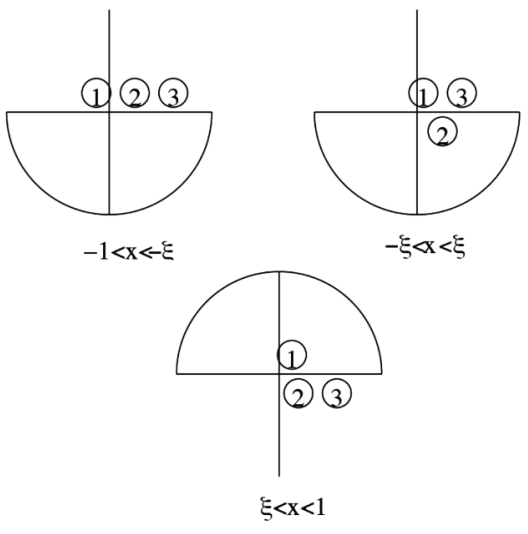

FIG. 8. Integration over the $\tilde{k}^{-}$variable in Eq. (78) is performed in the complex plane where location of the poles of the integrand is determined by the denominators in Eq. (79). Location of these poles is in agreement with Ref. [62]. For $x<$ $\xi, H(x<-\xi)$ receives a contribution from the $\hat{s}$-channel and for $x<\xi, H(x>\xi)$ is determined by the $\hat{u}$-channel.

which gives a positive (negative) definite $H$ function for $x>\xi$ (for $x<-\xi$ ) and the subscripts denote the value of $k^{-}=\kappa_{i}^{-}$obtained from the $i$ th pole. The perturbative scalar diquark model presented here is, by construction, "hard." That is, it incorporates the asymptotic behavior based on dimensional analysis, where $G_{M}(t) \propto 1 / t^{2}$, and it is difficult to reproduce the standard electromagnetic form factor at small momentum transfer. However, there exists a parameter set which correctly normalizes it to $F(0)=1$ and yields a Sachs form factor which is never further from data than by a factor 1.5-2. The parameters are $\Lambda=$ $0.8 \mathrm{GeV}, m_{q}=0.4 \mathrm{GeV}, g=25 \mathrm{GeV}$, and $\gamma=0.2$.

The resulting $1 / x$ form factor is shown in Fig. 9. To fix the isospin we have taken the rough approximation $H^{u}=$ $2 H^{d}$ and weighted each with $e_{q}^{2}$ as would be extracted from

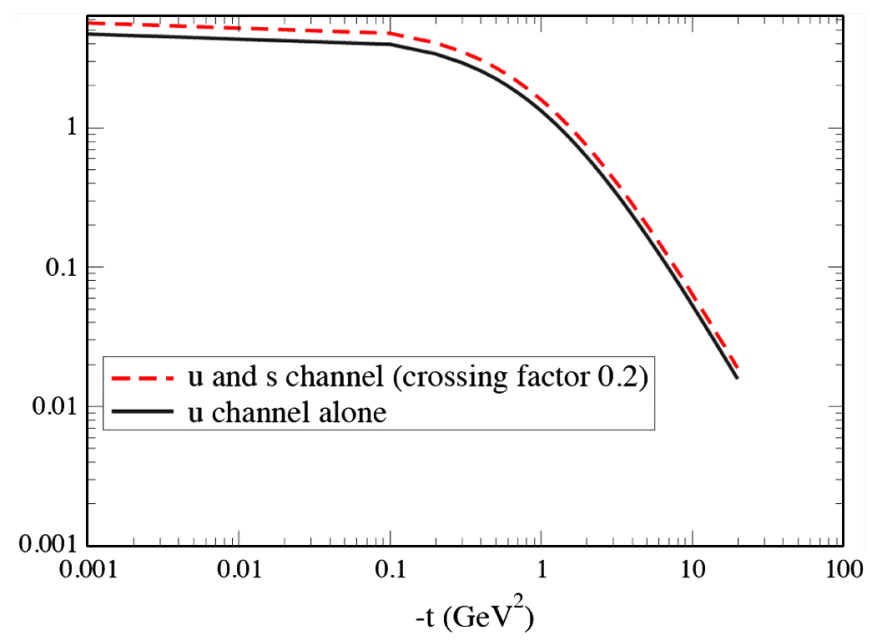

FIG. 9 (color online). The $F_{1 / x}$ form factor in the perturbative diquark model. At large $-t$ the form factor is power law suppressed.

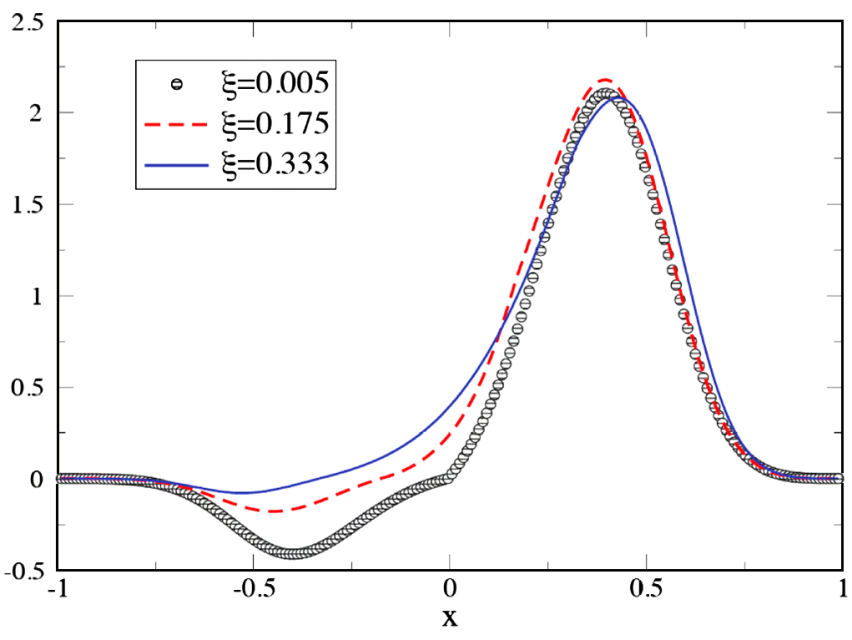

FIG. 10 (color online). The $H(x, \xi, t)$ GPD at fixed $-t=$ $1 \mathrm{GeV}^{2}$ with the parameters discussed in text.

DVCS. More discussion on isospin can be found in the appendix. In Fig. 10 we plot $H(x)$ varying $\xi$. For $x>0$, $H(x)$ describes valence quarks and yields a finite $1 / x$ form factor. For $x<0$ there is a small amount of antiquark sea (not Regge-dominated) which also contributes. The figure also illustrates that in this model $H(x)$ vanishes at break points, i.e. when $x \rightarrow \xi$. The perturbative diquark model can be used as a template to provide a general description of the parton-proton scattering amplitude by means of Regge and spectral analysis, as advanced by in Ref. [1,2].

\section{EXTRACTING THE $\boldsymbol{J}=0$ FIXED POLE FROM EXPERIMENTAL DATA}

Early analysis of SLAC and Daresbury data revealed a fixed pole in the real proton and neutron Compton amplitudes. The phenomenological analysis $[11,47]$ led to the following values for the forward limit of the $J=0$ pole amplitudes:

$$
\begin{aligned}
& T_{p}^{J=0}=-3(1) \frac{2 M_{N}}{\alpha_{\mathrm{em} .}} \mu b \mathrm{GeV}=-2.0(7), \\
& T_{n}^{J=0}=-0.5(1) \frac{2 M_{N}}{\alpha_{\mathrm{em} .}} \mu b \mathrm{GeV}=-0.3(7),
\end{aligned}
$$

for proton and neutron, respectively. Earlier evaluation by Creutz et al [48] yielded $T_{p}^{J=0}=-5(3)$ and it was observed that in magnitude and sign this amplitude is compatible with that of the Thomson term. It should be clear from the dispersion analysis, however, that this is a coincidence, and there is no reason why the amplitudes should be related.

A direct measurement of the fixed pole has to date not been performed. We now sketch how it can be extracted from deeply virtual Compton scattering. 


\section{A. Compton scattering}

We shall comment here on existing data on Compton scattering in regard to what they provide on the extraction of the $J=0$ pole. First let us note that the DVCS data obtained at HERA is well in the domain of Regge theory. At the stringently large $s$ and $Q^{2}$ required for the rest of its physics program, the experiments there could not reach large $-t$ due to statistical limitations and are therefore dominated by leading Regge exchanges. This is apparent in Fig. 11 where we plot $t$ dependence of the H1 data. Fast exponential falloff is observed. For comparison we also show the data for real Compton. This high-energy data has insufficient recoil, i.e. momentum transfer $t$, and is dominated by the Pomeron. Note that QCD counting rules [49] predict a power-law falloff for exclusive process and at fixed angle, for real Compton scattering, give

$$
\frac{d \sigma}{d t}=\frac{f\left(\theta_{\mathrm{CM}}\right)}{s^{6}} .
$$

However, the law is different and falls only as $s^{-2}$ in the Regge regime which has fixed $t$ as opposed to fixed scattering angle. This transition could be seen in the Compton data from the Cornell experiment and recent Jefferson Lab experiment, although typically perturbative QCD calculations are about an order of magnitude lower than the data interpreted as fixed-angle Compton scattering [50]. We shall now discuss $s$ dependence at fixed $t$. The prediction based on dominance of the $J=0$ pole is that the differential cross section, $d \sigma / d t$ should fall off as $s^{-2}$. This is not yet clear from the Cornell data, which we plot in Fig. 12. Ignoring the highest $s$-point it appears, however, the trend is correct and (in the log-log plot) the slope seems to soften with increasing $s$, showing the lessening influence of conventional Regge poles. Extending the kinematic region in either $t$ or $s$ should help isolate the asymptotic contribution expected to fall $s^{-2}$. The Jefferson Lab data at the highest $t$

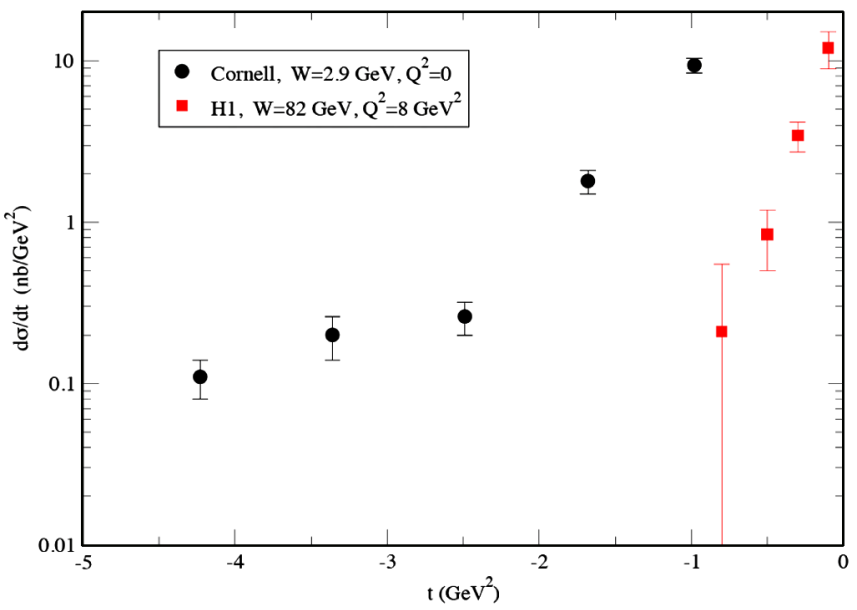

FIG. 11 (color online). The $t$ dependence of the real Compton and deeply virtual Compton scattering cross sections from Cornell [12] and H1 [63] experiments, respectively.

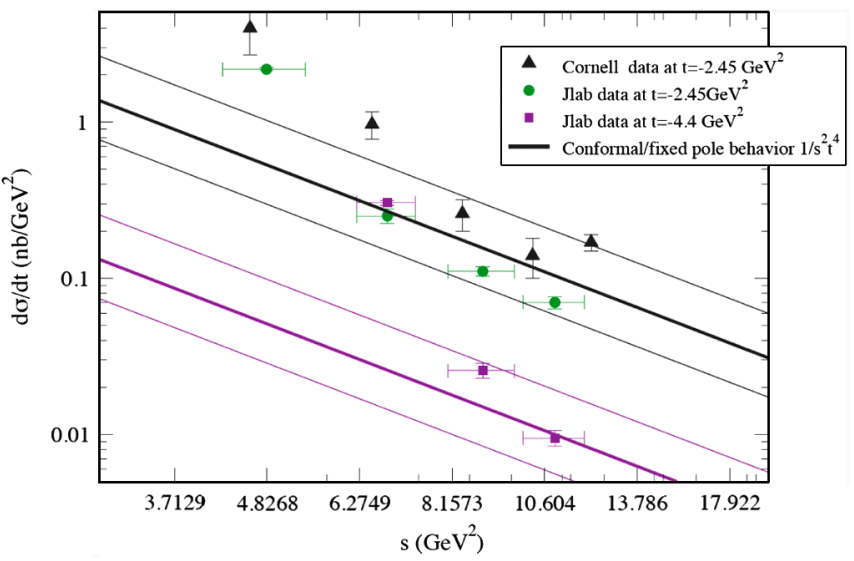

FIG. 12 (color online). The Cornell data from Ref. [12] is replotted for the fixed value of $t=-2.45 \mathrm{GeV}^{2}$. For comparison we also show recent Jefferson Lab data from Ref. [59].

clearly fails to be consistent with the fixed pole form alone, but the condition $s \gg-t$ is not well satisfied. At slightly lower $-t$, the data is consistent with the fixed-pole slope. The lines in the figure correspond to a fit to the form

$$
\frac{d \sigma_{\gamma p \rightarrow \gamma p}^{\mathrm{FP}}}{d t}=\frac{C^{\mathrm{FP}}}{s^{2} t^{4}}
$$

where the central line and error bands correspond to a value $\log C^{\mathrm{FP}}=6.1(6)$ with $C^{\mathrm{FP}}$ in units of $n b \mathrm{GeV}^{10}$.

The new H1 data at large energy [51], plotted in Fig. 13, is dominated by the pomeron even at their largest $-t=$ $0.8 \mathrm{GeV}^{2}$ bin (although the power-law exponent has already diminished considerably from its hard-pomeron value), and hence the fixed pole is not visible under this dominant Regge pole. Data at larger $-t$ is needed.

If we cross the Compton amplitude $\gamma p \rightarrow \gamma p$ we obtain the amplitude for proton-antiproton production by photofusion $\gamma \gamma \rightarrow p \bar{p}$ that has been studied in $e^{-} e^{+}$collider

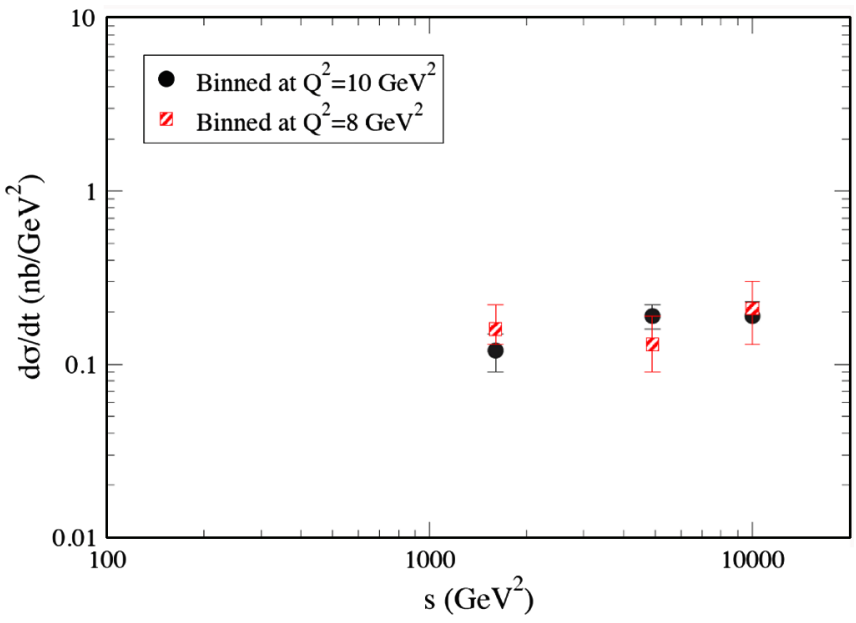

FIG. 13 (color online). The H1 data from Ref. [51] for $t=$ $-0.8 \mathrm{GeV}^{2}$ 
experiments L3 at LEP [52,53] and Belle at KEK [54] among others. At fixed angle and large $s, t$, photon-photon annihilation into mesons has been analyzed within the perturbative QCD framework [55-57]. The data, however, is again inconclusive in what pertains to the existence of the $J=0$ fixed pole.

In Ref. [55] it was shown that the photon-meson Compton scattering amplitude should have the $J=0$ pole behavior. In particular the Compton amplitude for vector mesons is found to be

$$
T_{\gamma V \rightarrow \gamma V}^{++}=16 \pi \alpha_{E M} F_{V}(t)\left(e_{1}^{2}+e_{2}^{2}\right) .
$$

However, the $J=0$ fixed pole decouples from pseudoscalar mesons, and it is not likely that this relation will be tested soon.

\section{B. What to expect from future measurements}

Given that the $J=0$ fixed pole cannot be claimed to have been conclusively extracted it would be very useful to have deeply virtual Compton scattering data for

$$
s \gg Q^{2} \gg-t>-t_{0} \sim 1 \mathrm{GeV}^{2} .
$$

Such kinematics is required for applicability of Regge and handbag-diagram phenomenology.

Currently, the HERA data does not satisfy the last inequality. Jefferson Lab with a $12 \mathrm{GeV}$ beam should be able to reach $s \simeq 40 \mathrm{GeV}^{2}, Q^{2} \simeq 6 \mathrm{GeV}^{2}, t \simeq-3 \mathrm{GeV}^{2}$ and be able to measure the virtual Compton amplitude where the $J=0$ pole dominates and extract its form factor. An electron-ion collider should be able to provide a definite measurement in the challenging kinematics required to extract the $J=0$ fixed pole and this adds to the further motivation of considering such a machine.

An important test of the handbag approximation and of whether the Compton amplitude is dominated by the $1 / x$ form factor is to measure the ratio of the differential cross sections on the neutron and on the proton, namely,

$$
R_{n / p}=\frac{\frac{d \sigma}{d t}(\gamma n \rightarrow \gamma n)}{\frac{d \sigma}{d t}(\gamma p \rightarrow \gamma p)} .
$$

Assuming isospin symmetry, that is, $H_{n}^{d}=H_{p}^{u}$, the ratio becomes

$$
R_{n / p}=\frac{\sum_{n} e_{q}^{2}}{\sum_{p} e_{q}^{2}}=\frac{2 e_{d}^{2}+e_{u}^{2}}{e_{d}^{2}+2 e_{u}^{2}}=\frac{2}{3},
$$

if both photons couple to a single quark, as in the handbag mechanism, (left diagram in Fig. 14), and smaller otherwise. In the extreme case of coherent scattering on valence quarks (right diagram in Fig. 14), the ratio is expected to be close to

$$
R_{n / p}=\frac{\sum_{n} e_{q_{i}} e_{q_{j}}}{\sum_{p} e_{q_{i}} e_{q_{j}}}=\frac{1}{3}
$$

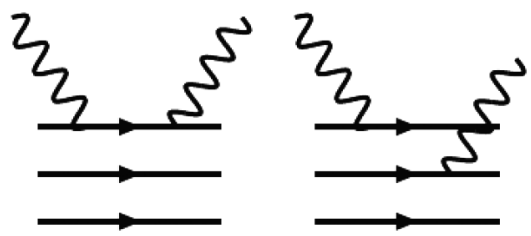

FIG. 14. In the handbag approximation incoherent scattering (left diagram) dominates over coherent processes with all quarks participating.

Another interesting measurement would be to follow the $t$ dependence of the Regge exponents, to ensure that indeed amplitudes at large momentum transfer $t$ can be understood as in Regge exchanges with intercepts $\alpha(t) \rightarrow-1$. Jefferson Lab could easily establish that the Reggeons recede below $\alpha=0$ in meson electroproduction, as the $t$ reach needed is only about $1 \mathrm{GeV}^{2}$. For each $t$ point, several $s$ measurements need to be taken with $s \gg-t$ to check the $s^{-\alpha(t)}$ law. As for $\rho_{L}^{0}$ electroproduction, there is abundant data on forward production at small and moderate $Q^{2}$ and $s$ (see the compilation in Ref. [58]) and larger $Q^{2}$ [42], but the extraction of the $J=-1 / 2$ analogous to the Compton fixed pole requires, as commented above, higher $t$.

\section{CONCLUSIONS}

The local coupling of two photons to the fundamental quark currents of a hadron gives an energy-independent contribution to the Compton amplitude proportional to the charge squared of the struck quark, a contribution which has no analog in hadron scattering reactions. The existence of this contribution, which is the analog of Thomson scattering on the electrons of an atom at high energies, provides a fundamental test of QCD. This paper is about the nucleon, but as we have discussed in the introduction, the fixed-pole concept is extensible to any bound system of pointlike (or effectively pointlike) charges. We have shown that this local contribution has a real phase and is universal, giving the same contribution for real or virtual Compton scattering for any photon virtuality and skewness at fixed momentum transfer squared $t$. The $t$ dependence of this $J=0$ fixed Regge pole is parameterized by a yet unmeasured even charge-conjugation form factor of the target nucleon. The $t=0$ limit gives an important constraint on the dependence of the nucleon mass on the quark mass through the Weisberger relation. Thus far, contemporary fits using conventional parton distributions have failed to unambiguously determine its value. Compton scattering of real photons at large $t$ is especially interesting because the $J=0$ fixed pole gives a purely real $s$ - and $Q^{2}$-independent amplitude.

The analysis of this paper provides a systematic procedure for identifying and verifying the $J=0$ fixed-pole contribution to real and virtual Compton scattering. First, one identifies a candidate $J=0$ contribution to the real 
Compton scattering cross section $d \sigma / d t(\gamma p \rightarrow \gamma p)$ at $s \gg-t$ which scales as $1 / s^{2}$ at fixed $t$; i.e., a contribution to the Compton cross section which scales as the elementary Klein-Nishina scattering cross section for $\gamma q \rightarrow \gamma q$ times the square of a form factor $F_{1 / x}(t)$. Such a contribution is possibly apparent in recent results from the E99-114 Hall A experiment at Jefferson Lab [59]. If this contribution is, in fact, due to the local coupling of the two photons to the quark, it will be independent of the photon virtuality $q^{2}$ at fixed $t$, when one measures high-energy virtual Compton scattering $\gamma^{*}(q) p \rightarrow \gamma p$. Since the $J=0$ amplitude is real, it will have maximum interference with the real Bethe-Heitler amplitude in $e p \rightarrow e p \gamma$. This program should be practical at the $12 \mathrm{GeV}$ Jefferson Laboratory facility.

We have also discussed how the $J=0$ fixed pole and the $1 / x$ form factor can be extracted from deeply virtual Compton scattering at large $t$ and have examined predictions given by models of the $H$ generalized parton distribution. The $J=0$ contributions are readily identifiable in DVCS at high $-t<0.6-1 \mathrm{GeV}^{2}$, where conventional Regge trajectories have receded. One can then test specific models such as the diquark model or quark model with light-front hadron wave functions, AdS/QCD predictions, and lattice calculations.

We also note that the $J=0$ fixed pole appears as a local energy-independent real contribution to the Compton amplitude for other two-photon processes such as the timelike real and virtual exclusive reactions $\gamma \gamma \rightarrow H \bar{H}, \gamma \gamma^{*} \rightarrow$ $H \bar{H}$, or $\bar{p} p \rightarrow H[60]$.

\section{ACKNOWLEDGMENTS}

We are indebted to many colleagues, among them Mischa Gorchteyn, Michael Peskin, Sidney Drell, Tim Londergan, Paul Hoyer, Anatoly Radyushkin, Ivan Schmidt, Dieter Mueller, and Markus Diehl, for useful conversations. F. J. L.-E. is grateful for the hospitality of the SLAC National Accelerator Laboratory theory group; the Indiana University Nuclear Theory Center where a sizeable part of this work was completed; partial financial support from a Fundacion del Amo-University; as well as Grants No. FPA 2004-02602, No. 2005-02327, No. PR27/ 05-13955-BSCH (Spain); and No. DE-FG0287ER40365 from the U.S. Department of Energy (USA)..

\section{APPENDIX A: ORIGIN OF THE $\boldsymbol{J}=0$ FIXED POLE: A SIMPLE MODEL}

In Regge theory, a Regge pole at $J=\alpha(t)$ (a singularity of the scattering matrix in the complex angular momentum plane) leads to a high-energy behavior of the scattering amplitude proportional to $s^{\alpha(t)}$ for $s \gg-t$. A $J=0$ pole thus corresponds to a scattering amplitude which is energy independent in the region of momentum transfer for which $\alpha(t)=0$. As we have noted in the introduction, this con-
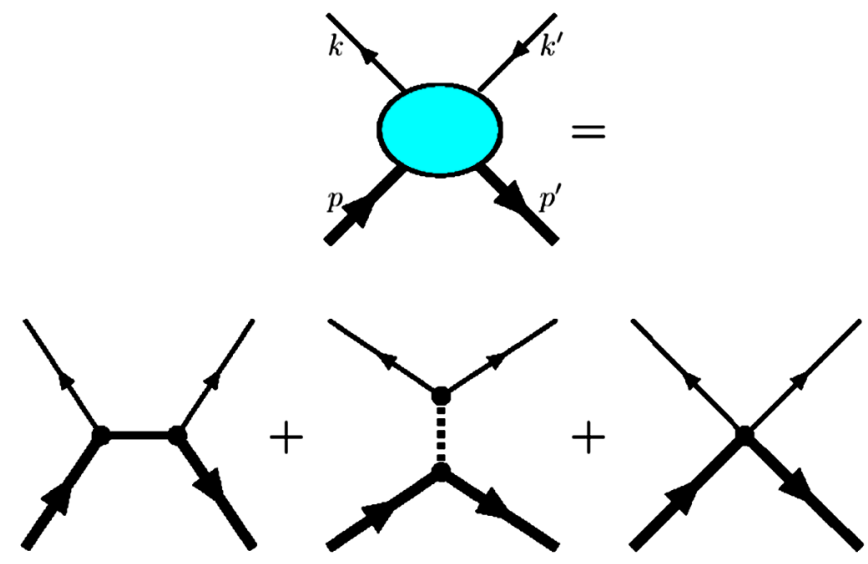

FIG. 15 (color online). A simple, perturbative model for a twobody scattering amplitude, given by the sum of an $s$-channel, $t$-channel, and contact interaction.

tribution is a fundamental prediction of QCD arising from the local two-photon interactions with the quark currents.

In perturbation theory, energy independence arises from contact interactions, as indicated by the last diagram in Fig. 15. To see this, consider an $s$-channel exchange (lower-left panel on Fig. 15) of a spin-0 particle of mass $M$ (for simplicity we ignore the natural width). The corresponding amplitude $A_{s}$ is proportional to

$$
A_{s}(s, M)=\frac{M^{2}}{M^{2}-s} .
$$

The two limits are interesting. If $s \rightarrow \infty$ at fixed $M$, one has $A_{s} \sim s^{-1}$, or $\alpha=-1$. We have normalized $A_{s}$ such that in the other limit, $M \rightarrow \infty$, which corresponds to a pointlike interaction with $\alpha=0, A_{s}$ remains finite. This $s$-channel exchange can be represented as an infinite series of $t$-channel exchanges of different spins. A standard way to expose this duality is to perform the Mellin transformation which enables one to write the amplitude in Eq. (A1) as

$$
A_{s}(s . M)=\frac{1}{2 \pi i} \int_{c-i \infty}^{c+i \infty} d \alpha \frac{\pi}{\sin \pi \alpha}\left(-\frac{M^{2}}{s}\right)^{\alpha},
$$

where $0<c<1$. For large c.m. energies $s>M^{2}$ the contour for the integral can be closed to encircle the positive real axis with $\alpha>c$ and then replaced by the sum over poles of $\sin \pi \alpha$ which occur at integer $\alpha=J$ with $J \geq 1$. The residues at these poles are

$$
\text { residue }_{\alpha=J}\left(\frac{\pi}{\sin \pi \alpha}\right)=(-1)^{J},
$$

and the amplitude becomes

$$
A_{s}\left(s>M^{2}\right)=\sum_{J \geq 1}(-1)^{J+1}\left(-\frac{s}{M^{2}}\right)^{-J} .
$$

For small c.m. energies, on the other hand, $s<M^{2}$ the contour can be closed and the integral replaced by a sum over poles to the left of the $\alpha=c$ line that occurs at integer 
$\alpha=-J$ including the $J=0$ pole,

$$
A_{s}\left(s<M^{2}, M\right)=\sum_{J \geq 0}(-1)^{J}\left(-\frac{s}{M^{2}}\right)^{J}
$$

[these relations are easy to check since they simply reconstruct as a geometric series Eq. (A1)]. The $s^{J}$ dependence of individual amplitudes on the right-hand side of Eq. (A5) is what is expected from exchange of a spin- $J$ object in the $t$-channel. The large-s behavior of the amplitude in Eq. (A1) then corresponds formally, in the $t$-channel, to a sum over exchanges of negative spin. Phenomenologically, hadron amplitudes with $J=\alpha<0$ indeed occur for physical $s$ and large and negative momentum transfer $t<t_{0}<0$ [16]. This is analogous to the simple model defined by the amplitude in Eq. (A1), in the language of Regge phenomenology, where the asymptotic behavior for $s \gg M^{2}$ would correspond to an exchange of $J=\alpha=-1 t$-channel trajectory. From Eq. (A5) it follows that the pointlike interaction, obtained in the limit $M^{2} \rightarrow \infty$, in the Regge language, corresponds to an exchange of an object with spin, $J=0$. We finally note that the presence of pointlike scattering is a necessary but not sufficient condition for the $J=0$ pole. A combination $A_{s}\left(s, M_{1}\right)-A_{s}\left(s, M_{2}\right)$ in the pointlike limit $s \ll M_{1}^{2}, M_{2}^{2}$ has a vanishing $J=0$ amplitude [6]. If either the parton-proton or parton-photon interactions have a pointlike contact interaction, this will survive convolution with the rest of the amplitude and reflect as a $J=0$ component of the photon-proton amplitude. Conversely, experimentally establishing this becomes a signature of pointlike scattering on underlying elementary constituents.

\section{APPENDIX B: ISOSPIN ANALYSIS}

Throughout the paper quark charges are measured in units of the electron charge. The quark flavor decomposition of the proton GPD is given by

$$
H_{F_{1}}^{p}=\sum e_{q} H^{q / p}=\frac{2}{3} H^{u / p}-\frac{1}{3} H^{d / p} .
$$

We ignore the quark sea, and assume the naive quark model assignment $p=u u d, n=u d d$, and therefore set $H^{u / p}=$ $2 H^{d / p}$ and neglect $H^{s / p}$. We will call $H^{d / p}$ simply $H$ and therefore

$$
H_{F_{1}}^{p}=H .
$$

In the case of the neutron, and profiting from isospin symmetry, we have

$$
H_{F_{1}}^{n}=\frac{2}{3} H^{u / n}-\frac{1}{3} H^{d / n}=\frac{2}{3} H^{d / p}-\frac{1}{3} H^{u / p}=0,
$$

which of course is expected to receive corrections from sea quarks. Turning to DVCS, the relevant combinations are now

$$
\begin{aligned}
& H_{\mathrm{DVCS}}^{p}=\sum e_{q}^{2} H^{q / p}=\frac{4}{9} H^{u / p}+\frac{1}{9} H^{d / p}=H, \\
& H_{\mathrm{DVCS}}^{n}=\frac{2}{3} .
\end{aligned}
$$

A simultaneous analysis of DVCS for the proton and the neutron allows the extraction of both $u$ and $d 1 / x$ moments

$$
\begin{aligned}
& \int \frac{d x}{x} H^{u}(x, 0, t)=\frac{3}{5}\left(4 F_{1 / x}^{p}(t)-F_{1 / x}^{n}(t)\right), \\
& \int \frac{d x}{x} H^{d}(x, 0, t)=\frac{3}{5}\left(-F_{1 / x}^{p}(t)+4 F_{1 / x}^{n}(t)\right),
\end{aligned}
$$

which extrapolated to $t \rightarrow 0$ can be compared with Eq. (58).
[1] S. J. Brodsky, F. E. Close, and J. F. Gunion, Phys. Rev. D 5, 1384 (1972).

[2] S. J. Brodsky, F. E. Close, and J. F. Gunion, Phys. Rev. D 6, 177 (1972).

[3] M. Diehl, Phys. Rep. 388, 41 (2003).

[4] K. J. Golec-Biernat and A. D. Martin, Phys. Rev. D 59, 014029 (1998).

[5] W. I. Weisberger, Phys. Rev. D 5, 2600 (1972).

[6] M. Creutz, Phys. Rev. D 7, 1539 (1973).

[7] S. J. Brodsky, H. C. Pauli, and S. S. Pinsky, Phys. Rep. 301, 299 (1998).

[8] S. J. Brodsky and J. R. Primack, Ann. Phys. (N.Y.) 52, 315 (1969).

[9] A. Danagulian, A. M. Nathan, M. Roedelbronn, D. J. Hamilton, C.E. Hyde, V.H. Mamian, and B. Wojtsekhowski, Nucl. Phys. A755, 281 (2005).

[10] S. J. Brodsky, F. E. Close, and J. F. Gunion, Phys. Rev. D 8, 3678 (1973).
[11] M. Damashek and F. J. Gilman, Phys. Rev. D 1, 1319 (1970).

[12] M. A. Shupe et al., Phys. Rev. D 19, 1921 (1979).

[13] C. R. Ji, Y. Mishchenko, and A. Radyushkin, Phys. Rev. D 73, 114013 (2006).

[14] A. Zee, Phys. Rev. D 5, 2829 (1972) 6, 938(E) (1972).

[15] S. J. Brodsky and I. A. Schmidt, Phys. Rev. D 43, 179 (1991).

[16] D. D. Coon, J.F. Gunion, J. Tran Thanh Van, and R. Blankenbecler, Phys. Rev. D 18, 1451 (1978).

[17] I. V. Anikin and O. V. Teryaev, Phys. Rev. D 76, 056007 (2007).

[18] M. Diehl and D. Yu. Ivanov, Eur. Phys. J. C 52, 919 (2007).

[19] M. V. Polyakov, Phys. Lett. B 659, 542 (2008).

[20] J. C. Collins, L. Frankfurt, and M. Strikman, Phys. Rev. D 56, 2982 (1997).

[21] J. C. Collins and A. Freund, Phys. Rev. D 59, 074009 
(1999).

[22] A.P. Szczepaniak, J. T. Londergan, and F. J. LlanesEstrada, arXiv:0707.1239.

[23] J. Kuti and V.F. Weisskopf, Phys. Rev. D 4, 3418 (1971).

[24] A. D. Martin, R. G. Roberts, W. J. Stirling, and R. S. Thorne, Eur. Phys. J. C 4, 463 (1998).

[25] M. Gluck, E. Reya, and A. Vogt, Eur. Phys. J. C 5, 461 (1998).

[26] R. Alkofer, P. Bicudo, S. R. Cotanch, C. S. Fischer, and F. J. Llanes-Estrada, arXiv:nucl-th/0601032.

[27] D. S. Armstrong et al. (G0 Collaboration), Phys. Rev. Lett. 95, 092001 (2005).

[28] P. A. Boyle et al., J. High Energy Phys. 07 (2008) 112.

[29] A. V. Radyushkin, Phys. Rev. D 59, 014030 (1998).

[30] M. V. Polyakov and C. Weiss, Phys. Rev. D 60, 114017 (1999).

[31] B. Lehmann-Dronke, A. Schaefer, M. V. Polyakov, and K. Goeke, Phys. Rev. D 63, 114001 (2001).

[32] I. V. Anikin, B. Pire, L. Szymanowski, O. V. Teryaev, and S. Wallon, Eur. Phys. J. C 42, 163 (2005).

[33] S. J. Brodsky, M. Diehl, and D.S. Hwang, Nucl. Phys. B596, 99 (2001).

[34] S. J. Brodsky and F. J. Llanes-Estrada, Eur. Phys. J. C 46, 751 (2006).

[35] M. Diehl, T. Feldmann, R. Jakob, and P. Kroll, Eur. Phys. J. C 8, 409 (1999).

[36] G. P. Lepage and S. J. Brodsky, Phys. Rev. Lett. 43, 545 (1979) 43, 1625(E) (1979).

[37] F. Schlumpf, J. Phys. G 20, 237 (1994).

[38] S. J. Brodsky and F. Schlumpf, Prog. Part. Nucl. Phys. 34, 69 (1995).

[39] G. F. de Teramond and S. J. Brodsky, Phys. Rev. Lett. 94, 201601 (2005).

[40] P. Hoyer, J. T. Lenaghan, K. Tuominen, and C. Vogt, Phys. Rev. D 70, 014001 (2004).

[41] A. Gardestig, A.P. Szczepaniak, and J. T. Londergan, Phys. Rev. D 68, 034005 (2003).

[42] M. Vanderhaeghen, P. A. M. Guichon, and M. Guidal,
Phys. Rev. D 60, 094017 (1999).

[43] M. Diehl, T. Feldmann, R. Jakob, and P. Kroll, Eur. Phys. J. C 39, 1 (2005).

[44] P. V. Landshoff, J. C. Polkinghorne, and R. D. Short, Nucl. Phys. B28, 225 (1971).

[45] A. Van Dyck, T. Van Cauteren, and J. Ryckebusch, arXiv:0710.2271.

[46] Note the typographical error in Ref. [34].

[47] C. A. Dominguez, J. F. Gunion, and R. Suaya, Phys. Rev. D 6, 1404 (1972).

[48] M. J. Creutz, S. D. Drell, and E. A. Paschos, Phys. Rev. 178, 2300 (1969).

[49] S. J. Brodsky and G. R. Farrar, Phys. Rev. D 11, 1309 (1975).

[50] T. C. Brooks and L. J. Dixon, Phys. Rev. D 62, 114021 (2000).

[51] F. D. Aaron et al. (H1 Collaboration), Phys. Lett. B 659, 796 (2008).

[52] P. Achard et al. (L3 collaboration), Phys. Lett. B 571, 11 (2003).

[53] T. Barillari, arXiv:hep-ex/0603019.

[54] C. C. Kuo et al. (Belle Collaboration), Phys. Lett. B 621, 41 (2005).

[55] S. J. Brodsky and G. P. Lepage, Phys. Rev. D 24, 1808 (1981).

[56] M. Anselmino, F. Caruso, P. Kroll, and W. Schweiger, Int. J. Mod. Phys. A 4, 5213 (1989).

[57] H. W. Huang, P. Kroll, and T. Morii, Eur. Phys. J. C 23, 301 (2002) 31, 279(E) (2003).

[58] T. H. Bauer, R. D. Spital, D. R. Yennie, and F. M. Pipkin, Rev. Mod. Phys. 50, 261 (1978) 51, 407(E) (1979).

[59] A. Danagoulian et al. (Hall A Collaboration), Phys. Rev. Lett. 98, 152001 (2007).

[60] J. Pinfold, CERN Courier 48, 23 (2008).

[61] E. J. Brash, A. Kozlov, S. Li, and G. M. Huber, Phys. Rev. C 65, 051001 (2002).

[62] M. Diehl and T. Gousset, Phys. Lett. B 428, 359 (1998).

[63] A. Aktas et al. (H1 Collaboration), Eur. Phys. J. C 44, 1 (2005). 